\title{
Primordial non-Gaussianities in single field inflationary models with non-trivial initial states
}

\author{
Sina Bahrami and Éanna É. Flanagan \\ Department of Physics, Cornell University, Ithaca, NY 14853, USA. \\ Draft of August 19, 2014; printed June 27, 2018 at 4:59 \\ E-mail: sb933@cornell.edu, eef3@cornell.edu
}

\begin{abstract}
We compute the non-Gaussianities that arise in single field, slow roll inflationary models arising from arbitrary homogeneous initial states, as well as subleading contributions to the power spectrum. Non Bunch-Davies vacuum initial states can arise if the transition to the single field, slow roll inflation phase occurs only shortly before observable modes left the horizon. They can also arise from new physics at high energies that has been integrated out. Our general result for the bispectrum exhibits several features that were previously seen in special cases.
\end{abstract}




\section{Contents}

1 Introduction and summary $\quad 1$

1.1 Background and motivation 1

1.2 Summary of results 3

2 Review of basic results in single field inflationary models 4

2.1 Generation of perturbations neglecting interactions 4

2.2 Including the leading order effects of interactions 6

3 Choice of initial state $\quad 6$

3.1 Parameterization of general homogeneous states 6

3.2 Constraints from backreaction considerations 8

$\begin{array}{llr}\text { 3.2.1 Constraints under conservative assumptions } & 9\end{array}$

3.2.2 Constraints under more speculative assumptions 11

4 Scalar power spectrum $\quad 12$

$\begin{array}{lll}4.1 & \text { Leading order power spectrum } & 12\end{array}$

4.2 Subleading contributions to power spectrum due to interactions 13

5 Primordial non-Gaussianities: bispectrum of scalar perturbations $\quad 14$

$\begin{array}{lll}5.1 & \text { Vacuum contribution } & 15\end{array}$

5.2 Contribution from two point function 15

$\begin{array}{lll}5.3 & \text { Contribution from three point function } & 17\end{array}$

5.4 Contribution from connected piece of four point function 17

6 Order of magnitude estimates and discussion $\quad 18$

$\begin{array}{ll}6.1 \text { Generic initial states } & 19\end{array}$

6.2 Initial states with vanishing three point function 20

$\begin{array}{ll}\text { 6.2.1 Large occupation number regime } & 20\end{array}$

$\begin{array}{lll}6.2 .2 & \text { Limiting shape regimes } & 21\end{array}$

6.2.3 Observational constraints 23

6.3 Example: multimode squeezed initial states 24

7 Acknowledgments $\quad 24$

$\begin{array}{ll}\text { A Free functions for the dressed vacuum state } & 24\end{array}$

B Explicit calculation of the bispectrum $\quad 26$

B.1 Field redefinition contribution 26

\begin{tabular}{ll} 
B.2 Commutator contribution & 27 \\
\hline
\end{tabular}

\section{Introduction and summary}

\subsection{Background and motivation}

Inflationary models are in good quantitative agreement with data obtained from the cosmic microwave background (CMB) and large scale structure [1-8]. Present experimental data 
already rule out some inflationary models and constrain others. The CMB temperature inhomogeneities are distributed following a nearly scale-invariant spectrum, which is consistent with the predictions of inflation [9-13]. In addition, one of the key predictions of inflation is that the statistical properties of the fluctuations are Gaussian to high accuracy, which has been confirmed by observations, most recently by the Planck satellite [8]. However, small amounts of non-Gaussianity are predicted to be present, with a size that depends on the details of the model, and it is hoped to use future observations of non-Gaussianity to differentiate between different models [14].

The robustness of the predictions of inflation depends in part on the assumption that inflation started considerably before the horizon exit of the largest modes we can observe today. This occurs in any model where the total number $N$ of $e$-folds of inflation is significantly larger than the minimum number $N_{\min } \approx 60$ required to solve the horizon and flatness problems. In many models $N \gg N_{\min }$ is very natural, while $N \approx N_{\min }+$ (a few) is somewhat unnatural or fine tuned. Nevertheless it is still interesting to consider the possibility that $N \approx N_{\min }+$ (a few), since it opens up a richer set of possibilities for inflationary predictions. In particular, the well known statistical anomalies of low multipoles of the CMB, as recently confirmed and extended by Planck [15], may be a hint in this direction.

When the epoch of inflation that we can probe observationally occurs shortly after the start of inflation, there are a number of different non-standard effects that can arise. One is non-isotropy of the geometry: the initial geometry can have a Kasner-like anisotropic component that leaves an imprint on the perturbations at late times. The leading order effect on the power spectrum in these models can be written as

$$
P(\vec{k})=P_{0}(k)+P_{1}(k) Q_{i j} \hat{k}^{i} \hat{k}^{j},
$$

where $\vec{k}$ is a spatial wavevector, $k=|\vec{k}|$ is its magnitude and $\hat{k}^{i}=k^{i} / k$. Here $Q_{i j}$ is a symmetric traceless tensor which singles out preferred directions in space associated with the anisotropy at early times, and the functions $P_{0}$ and $P_{1}$ are functions of $k$ only. These models have been studied by Refs. [16-23]. However, the recent Planck data show no statistically significant quadrupolar component of the form (1.1) [see Fig. 34 of Ref. [15]].

A second type of effect that can occur when inflation starts just before the epoch we observe is non Bunch-Davies vacuum initial states. These can arise from effects at earlier times that are outside the domain of validity of a single field model, for example multifield effects (see eg. Ref. [24]). The choice of initial state is constrained somewhat by the requirement that backreaction due to the energy density and pressure of the initial state must be a small perturbation to the inflationary background, in order to have a self consistent computational framework, see section 3.2 below.

Non Bunch-Davies initial states can modify both the spectrum and bispectrum of the perturbations, and in particular can enhance the size of the bispectrum. Non-Gaussianities arising from specific classes of nontrivial initial states have been studied by many authors, including particle number eigenstates [25], Gaussian states [26], general multimode squeezed (i.e. vacuum) states [27-35], coherent states [36], thermal states [37], and so-called "calm states" [38]. In this paper we shall generalize these results to arbitrary initial states that are homogeneous. As first pointed out by Agullo and Parker [25], the squeezed triangle limit of the bispectrum can be significantly enhanced over its Bunch-Davies value; this remains true for the more general initial states analyzed here.

A second motivation for considering non Bunch-Davies vacuum initial states, separate from the possibility of inflation starting shortly before the epoch probed by current observa- 
tions, is provided by the trans-Planckian issue [39-49]. Modes that we observe today have physical momenta $k_{\text {phys }}=k / a$ which exceeds the Planck scale $M_{p}$ at sufficiently early times. A complete description of the physics at that stage will only be possible once we have a solid understanding of quantum gravity. For a given mode, the quantum gravitational corrections to the dynamics at horizon crossing are suppressed by $\sim H^{2} / M_{p}^{2}$ if the evolution is always adiabatic, where $H$ is the Hubble parameter during inflation, but could be larger and scale as $\sim H / M_{p}$ if violations of adiabaticity occur. In many toy models of quantum gravity effects, the effects of the Planck scale corrections to the dynamics of the modes can be mimicked by using the standard dynamics but with a modified initial state. Many computations have been performed of modifications to the spectrum and bispectrum of the perturbations due to new physics at high energies [34, 42-49]. The general results of this paper could be used as a tool in such computations.

\subsection{Summary of results}

In this paper we start with a general mixed quantum state describing a statistical distribution of initial inflaton perturbations, constrained only by the assumption of spatial homogeneity. We calculate the scalar power spectrum and bispectrum of the comoving curvature perturbation $\mathcal{R}$. Our general result for the bispectrum, given in Eq. (5.3) below, exhibits several features that were previously seen in special cases:

- The three point function of the initial state will generically ${ }^{1}$ give the dominant contribution to the bispectrum, since the contributions from the initial two and four point functions are suppressed by the factor $\sim H \sqrt{\epsilon} / M_{p}$, where $H$ is the inflationary Hubble scale, $M_{p}$ is the Planck scale, and $\epsilon$ is a slow roll parameter. This dominance of the three point function was previously seen in a special class of states by Agarwal et al. [26]. It is easy to understand: a nonzero bispectrum is obtained from an initial three point function from just the linear evolution, whereas contributions from the initial two and four point functions require nonlinearities. The bispectrum can be significantly larger than the Bunch-Davies bispectrum, cf. Eq. (6.4) below, even when initial occupation numbers are small and corrections to the power spectrum [Eq. (4.6) below] are small.

- For initial states with vanishing three point function (the subject of most previous studies), the bispectrum can be enhanced compared to the Bunch-Davies bispectrum in the squeezed triangle limit, as discovered by Agullo and Parker [25] for initial states consisting of statistical mixtures of occupation number eigenstates. For initial occupation numbers of order unity, the enhancement factor is the ratio of wavenumbers of the large scale mode and the small scale mode [Eq. (6.9) below]. We argue that backreaction considerations limit the enhancement factor to be $\lesssim 200$ (Sec. 6.2.2).

- For initial states with vanishing three point function, the bispectrum can be enhanced compared to the Bunch-Davies bispectrum in the elongated triangle limit, as discovered by Chen et al. [57] and Holman and Tolley [29] for generalized vacuum states (multimode squeezed states), and studied extensively by Agullo and Shandera [33]. We argue that the enhancement factor is limited by backreaction considerations to be of the same order as the enhancement factor of the squeezed triangle limit or smaller (Sec. 6.2.2).

\footnotetext{
${ }^{1}$ By "generic" we mean that there is no suppression of the initial three point function compared to the initial two and four point functions. This can be violated in specific scenarios, as discussed further in Sec. 6.1 below.
} 
- The bispectrum for non-vacuum initial states can violate the consistency relation of Creminelli and Zaldarriaga [50] for the squeezed triangle limit, which assumes an initial Bunch-Davies vacuum, as noted previously in Refs. [26, 33].

\section{Review of basic results in single field inflationary models}

\subsection{Generation of perturbations neglecting interactions}

The action for single field slow-roll models of inflation is

$$
S=\frac{1}{2} \int d^{4} x \sqrt{-g}\left[M_{p}^{2} R-(\nabla \phi)^{2}-2 V(\phi)\right]
$$

where $R$ is the Ricci curvature, $\phi$ is the inflaton field, and we use units with $c=\hbar=1$. We consider linear scalar perturbations about a background Friedmann-Robertson-Walker metric in comoving gauge, where

$$
\begin{aligned}
d s^{2} & =-(1+2 \Phi) d t^{2}+a(t)^{2}(1-2 \Psi) d x^{2}+2 a(t) B_{, i} d x^{i} d t, \\
\phi(t, \vec{x}) & =\phi(t)+\delta \phi(t, \vec{x}),
\end{aligned}
$$

with $\delta \phi(t, \vec{x})=0[3]$. Here $a(t)$ is the scale factor and $\Phi, \Psi$, and $B$ are scalar potentials. The background equations of motion are the Friedmann equations

$$
3 M_{p}^{2} H^{2}=\frac{\dot{\phi}^{2}}{2}+V(\phi), \quad \dot{H}=-\frac{1}{2 M_{p}^{2}} \dot{\phi}^{2},
$$

where $H \equiv \dot{a} / a$ is the Hubble parameter, and the Klein Gordon equation

$$
\ddot{\phi}+3 H \dot{\phi}+V^{\prime}(\phi)=0
$$

The potential slow roll parameters $\epsilon$ and $\eta$ are defined by

$$
\epsilon=\frac{M_{p}^{2}}{2}\left(\frac{V^{\prime}}{V}\right)^{2} \approx \frac{\dot{\phi}^{2}}{2 H^{2} M_{p}^{2}}, \quad \eta=M_{p}^{2}\left(\frac{V^{\prime \prime}}{V}\right) \approx-\frac{\ddot{\phi}}{H \dot{\phi}}+\frac{\dot{\phi}^{2}}{2 H^{2} M_{p}^{2}},
$$

where prime denotes differentiation with respect to the field $\phi$, and the approximate equalities are valid in the field slow roll limit.

We define a gauge invariant quantity called the comoving curvature perturbation $\mathcal{R}$ by

$$
\mathcal{R}=\Psi+\frac{H}{\dot{\phi}} \delta \phi
$$

In our choice of gauge we have $\mathcal{R}=\Psi$. To find the leading order action describing the evolution of $\mathcal{R}$ we expand the action (2.1) to second order in $\mathcal{R}=\Psi, B$ and $\Phi$, integrate out the non-dynamical fields $\Phi$ and $B$, and simplify using the background equations of motion and using integrations by parts. The final result is [3]

$$
S_{2}=\frac{1}{2} \int d^{3} x \int d \tau z^{2}\left[\mathcal{R}^{\prime 2}-(\partial \mathcal{R})^{2}\right],
$$


where $\tau \equiv \int d t / a(t)$ is the conformal time, $z \equiv a \dot{\phi} / H$, primes denote derivatives with respect to $\tau$, and $(\partial \mathcal{R})^{2} \equiv \delta^{i j} \mathcal{R}_{, i} \mathcal{R},{ }_{j}$. We decompose the curvature perturbation into spatial Fourier modes via

$$
\mathcal{R}(\vec{x}, \tau)=\int \frac{d^{3} k}{(2 \pi)^{3}} \mathcal{R}_{\vec{k}}(\tau) e^{i \vec{k} \cdot \vec{x}} .
$$

Using the decomposition (2.8) in the equation of motion obtained from the action (2.7) yields the Mukhanov-Sasaki equation

$$
\mathcal{R}_{\vec{k}}^{\prime \prime}(\tau)+\frac{2}{z} z^{\prime} \mathcal{R}_{\vec{k}}^{\prime}(\tau)+k^{2} \mathcal{R}_{\vec{k}}(\tau)=0
$$

In the slow-roll limit, we have $z^{-1} z^{\prime} \approx(1+3 \epsilon-\eta) / \tau$.

We now quantize the theory by promoting $\mathcal{R}_{\vec{k}}(\tau)$ to an operator, $\mathcal{R}_{\vec{k}}(\tau) \rightarrow \hat{\mathcal{R}}_{\vec{k}}(\tau)$. Next for each $k$ we choose any complex solution $\mathcal{R}(k, \tau)$ of the Mukhanov-Sasaki equation (2.9), for which $\mathcal{R}(k, \tau)$ and $\mathcal{R}(k, \tau)^{*}$ form a basis of the two dimensional solution space and for which the quantity (2.11) below is positive. Since the operator $\hat{\mathcal{R}}_{\vec{k}}$ satisfies the Mukhanov-Sasaki equation we can decompose it on this basis:

$$
\hat{\mathcal{R}}_{\vec{k}}(\tau)=\hat{A}_{\vec{k}} \mathcal{R}(k, \tau)+\hat{A}_{-\vec{k}}^{\dagger} \mathcal{R}(k, \tau)^{*},
$$

where $\hat{A}_{\vec{k}}$ and $\hat{A}_{\vec{k}}^{\dagger}$ are annihilation and creation operators. We normalize the mode functions by requiring

$$
i z^{2}\left(\mathcal{R}^{*} \mathcal{R}^{\prime}-\mathcal{R}^{*} \mathcal{R}\right)=1
$$

It then follows from Eq. (2.7) that $\left[\hat{A}_{\vec{k}}, \hat{A}_{\vec{k}^{\prime}}^{\dagger}\right]=(2 \pi)^{3} \delta\left(\vec{k}-\vec{k}^{\prime}\right)$.

This construction allows some freedom in the choice of mode function $\mathcal{R}(k, \tau)$, which can be resolved by specifying a boundary condition at early times. The choice of mode function determines a corresponding choice of a vacuum state $|0\rangle$ for which $\hat{A}_{\vec{k}}|0\rangle=0$. The standard choice of vacuum is known as the Bunch-Davies vacuum [51], which is the Minkowski vacuum of a comoving observer in the distant past when the mode is deep inside the horizon. This choice of vacuum corresponds to the following boundary condition on the mode function [3]

$$
\mathcal{R}(k, \tau) \rightarrow \frac{e^{-i k \tau}}{z(\tau) \sqrt{2 k}}, \quad \text { for }|k \tau| \gg 1
$$

With this in hand and assuming the slow-roll parameters to be constant, we can find the unique solution to equation (2.9) [3]

$$
\mathcal{R}(k, \tau)=\left(\frac{-\pi \tau}{4 z^{2}}\right)^{1 / 2} H_{\mu}^{(1)}(-\tau k),
$$

where $H_{\mu}^{(1)}$ is a Hankel function with index $\mu=3 / 2+2 \epsilon-\eta$. In the superhorizon regime $|\tau k| \ll 1$, and assuming $\epsilon \ll 1$ and $\eta \ll 1$, the mode function becomes

$$
\mathcal{R}(k, \tau) \rightarrow i\left[\frac{1}{4 k^{3} \epsilon\left(\tau_{k}\right)}\right]^{1 / 2} \frac{H\left(\tau_{k}\right)}{M_{p}} .
$$

Here $\tau_{k}$ is the value of conformal time at which the mode $k$ exits the horizon, given by $k=a\left(\tau_{k}\right) H$. 


\subsection{Including the leading order effects of interactions}

The leading order nonlinearities for inflaton perturbations were first worked out in detail by Maldacena [52]. The interaction Hamiltonian is obtained by expanding the action (2.1) to third order in $\mathcal{R}$ and integrating out the non-dynamical fields (the lapse and the shift). Maldacena obtained a simple form for the interaction Hamiltonian by writing it in terms of a redefined curvature field $\mathcal{R}_{c}$ which is given by

$$
\mathcal{R}=\mathcal{R}_{c}+\left[\frac{1}{2} \frac{\ddot{\phi}}{\dot{\phi} H}+\frac{1}{8 M_{p}^{2}} \frac{\dot{\phi}^{2}}{H^{2}}\right] \mathcal{R}_{c}^{2}+\frac{1}{4 M_{p}^{2}} \frac{\dot{\phi}^{2}}{H^{2}} \partial^{-2}\left(\mathcal{R}_{c} \partial^{2} \mathcal{R}_{c}\right)
$$

Expanding the action (2.1) around the spatially homogeneous background solution to third order in a specific choice of gauge, Maldacena found

$$
S[\mathcal{R}]=S_{2}\left[\mathcal{R}_{c}\right]+S_{3}\left[\mathcal{R}_{c}\right]+\ldots,
$$

where the functional $S_{2}$ is the quadratic action (2.7) evaluated at $\mathcal{R}=\mathcal{R}_{c}$, and $S_{3}$ is given by

$$
S_{3}\left(\mathcal{R}_{c}\right)=-\int d \tau H_{\mathrm{int}}=\int d \tau \int d^{3} x a(\tau)^{3}\left(\frac{\dot{\phi}}{H}\right)^{4} H M_{p}^{-2} \mathcal{R}_{c}^{\prime 2} \partial^{-2} \mathcal{R}_{c}^{\prime} .
$$

Here as before primes denote differentiation with respect to $\tau$, and $H_{\text {int }}$ is the interaction Hamiltonian. We will use this interaction Hamiltonian and the field redefinition (2.15) to compute corrections to the spectrum in section 4 below, and to compute the bispectrum in section 5 below, for general initial states.

In the remainder of this paper, we shall perform computations using the redefined field $\mathcal{R}_{c}$, and use the field redefinition only to compute the observable quantities that are defined in terms of $\mathcal{R}$. In particular, the mode creation and annihilation operators will from now on be defined in terms of a mode expansion of the field $\hat{\mathcal{R}}_{c}$ :

$$
\hat{\mathcal{R}}_{c, \vec{k}}(\tau)=\hat{A}_{\vec{k}} \mathcal{R}(k, \tau)+\hat{A}_{-\vec{k}}^{\dagger} \mathcal{R}(k, \tau)^{*} .
$$

\section{Choice of initial state}

\subsection{Parameterization of general homogeneous states}

In this paper we will allow arbitrary homogeneous initial states, a class of states more general than that considered by Agullo and Parker [25], who assumed statistical mixtures of particle number eigenstates. We now turn to a description of how the initial state is parameterized.

For an initial density matrix $\hat{\rho}$, the spectrum and bispectrum of $\mathcal{R}$ at late times will be determined by the initial two point, three point, and four point functions at some initial conformal time $\tau_{0},\left\langle\hat{A}_{\vec{k}_{1}}^{\alpha} \hat{A}_{\vec{k}_{2}}^{\beta}\right\rangle,\left\langle\hat{A}_{\vec{k}_{1}}^{\alpha} \hat{A}_{\vec{k}_{2}}^{\beta} \hat{A}_{\vec{k}_{3}}^{\gamma}\right\rangle$, and $\left\langle\hat{A}_{\vec{k}_{1}}^{\alpha} \hat{A}_{\vec{k}_{2}}^{\beta} \hat{A}_{\vec{k}_{3}}^{\gamma} \hat{A}_{\vec{k}_{4}}^{\delta}\right\rangle$. Here $\langle\ldots\rangle$ means the expectation value $\operatorname{tr}\left[\hat{\rho}_{\ldots} ..\right]$, and $\hat{A}_{\vec{k}}^{\alpha}$ means either $\hat{A}_{\vec{k}}($ for $\alpha=0)$ or $\hat{A}_{\vec{k}}^{\dagger}($ for $\alpha=1)$.

We assume that the initial state is homogeneous. It follows that the initial two point function can be parameterized in terms of two functions $\mathcal{F}_{1}(\vec{k})$ and $\mathcal{F}_{2}(\vec{k})$ by

$$
\begin{aligned}
\left\langle\hat{A}_{\vec{k}_{1}} \hat{A}_{\vec{k}_{2}}\right\rangle & =(2 \pi)^{3} \delta\left(\vec{k}_{1}+\vec{k}_{2}\right) \mathcal{F}_{1}\left(\vec{k}_{1}\right), \\
\left\langle\hat{A}_{-\vec{k}_{1}}^{\dagger} \hat{A}_{\vec{k}_{2}}\right\rangle & =(2 \pi)^{3} \delta\left(\vec{k}_{1}+\vec{k}_{2}\right) \mathcal{F}_{2}\left(\vec{k}_{1}\right) .
\end{aligned}
$$


It is clear that $\mathcal{F}_{2}$ is real and $\mathcal{F}_{1}$ is even. Under translation $\vec{x} \rightarrow \vec{x}+\vec{a}$ we have $\hat{A}_{\vec{k}} \rightarrow \hat{A}_{\vec{k}} e^{-i \vec{k} \cdot \vec{a}}$, which dictates the appearance of the specific delta functions in Eqs. (3.1). For an isotropic initial state $\mathcal{F}_{1}$ and $\mathcal{F}_{2}$ are functions just of $k$.

Similarly, the three point function under the assumption of homogeneity can be parameterized in terms of two functions $\mathcal{H}_{1}\left(\vec{k}_{1}, \vec{k}_{2}\right)$ and $\mathcal{H}_{2}\left(\vec{k}_{1}, \vec{k}_{2}\right)$, defined by

$$
\begin{aligned}
\left\langle\hat{A}_{\vec{k}_{1}} \hat{A}_{\vec{k}_{2}} \hat{A}_{\vec{k}_{3}}\right\rangle & =(2 \pi)^{3} \delta\left(\vec{k}_{1}+\vec{k}_{2}+\vec{k}_{3}\right) \mathcal{H}_{1}\left(\vec{k}_{1}, \vec{k}_{2}\right), \\
\left\langle\hat{A}_{-\vec{k}_{3}}^{\dagger} \hat{A}_{\vec{k}_{1}} \hat{A}_{\vec{k}_{2}}\right\rangle & =(2 \pi)^{3} \delta\left(\vec{k}_{1}+\vec{k}_{2}+\vec{k}_{3}\right) \mathcal{H}_{2}\left(\vec{k}_{1}, \vec{k}_{2}\right) .
\end{aligned}
$$

Both $\mathcal{H}_{1}$ and $\mathcal{H}_{2}$ are symmetric under interchange of their arguments. Also the other expectation values $\left\langle\hat{A}_{\vec{k}_{1}}^{\alpha} \hat{A}_{\vec{k}_{2}}^{\beta} \hat{A}_{\vec{k}_{3}}^{\gamma}\right\rangle$ can be expressed in terms of $\mathcal{H}_{1}$ and $\mathcal{H}_{2}$. In the isotropic case, $\mathcal{H}_{1}$ and $\mathcal{H}_{2}$ depend only on $k_{1}, k_{2}$ and on the angle between $\vec{k}_{1}$ and $\vec{k}_{2}$. Equivalently, they are functions of $k_{1}, k_{2}$ and $k_{3}$, or of $k, k_{1} / k_{3}$ and $k_{2} / k_{3}$, where $k \equiv\left(k_{1} k_{2} k_{3}\right)^{1 / 3}$.

Finally, the initial 4-point function can be parameterized by three functions $\mathcal{G}_{1}, \mathcal{G}_{2}$, and $\mathcal{G}_{3}$ of 3 vectors:

$$
\begin{aligned}
\left\langle\hat{A}_{\vec{k}_{1}} \hat{A}_{\vec{k}_{2}} \hat{A}_{\vec{k}_{3}} \hat{A}_{\vec{k}_{4}}\right\rangle & =(2 \pi)^{6} \delta\left(\vec{k}_{1}+\vec{k}_{2}+\vec{k}_{3}+\vec{k}_{4}\right) \mathcal{G}_{1}\left(\vec{k}_{1}, \vec{k}_{2}, \vec{k}_{3}\right), \\
\left\langle\hat{A}_{-\vec{k}_{1}}^{\dagger} \hat{A}_{\vec{k}_{2}} \hat{A}_{\vec{k}_{3}} \hat{A}_{\vec{k}_{4}}\right\rangle & =(2 \pi)^{6} \delta\left(\vec{k}_{1}+\vec{k}_{2}+\vec{k}_{3}+\vec{k}_{4}\right) \mathcal{G}_{2}\left(\vec{k}_{1}, \vec{k}_{2}, \vec{k}_{3}\right), \\
\left\langle\hat{A}_{-\vec{k}_{1}}^{\dagger} \hat{A}_{-\vec{k}_{2}}^{\dagger} \hat{A}_{\vec{k}_{3}} \hat{A}_{\vec{k}_{4}}\right\rangle & =(2 \pi)^{6} \delta\left(\vec{k}_{1}+\vec{k}_{2}+\vec{k}_{3}+\vec{k}_{4}\right) \mathcal{G}_{3}\left(\vec{k}_{1}, \vec{k}_{2}, \vec{k}_{3}\right) .
\end{aligned}
$$

These functions inherit some symmetry properties from their definitions. The function $\mathcal{G}_{1}$ is symmetric in all three of its arguments, and in addition obeys the identity

$$
\mathcal{G}_{1}\left(\vec{k}_{1}, \vec{k}_{2}, \vec{k}_{3}\right)=\mathcal{G}_{1}\left(\vec{k}_{1}, \vec{k}_{2},-\vec{k}_{1}-\vec{k}_{2}-\vec{k}_{3}\right),
$$

which follows from the invariance of the left hand side of Eq. (3.3a) under $\vec{k}_{3} \leftrightarrow \vec{k}_{4}$. Similarly the function $\mathcal{G}_{2}$ is symmetric in it second and third argument, and obeys the identities

$$
\begin{aligned}
& \mathcal{G}_{2}\left(\vec{k}_{1}, \vec{k}_{2}, \vec{k}_{3}\right)=\mathcal{G}_{2}\left(\vec{k}_{1},-\vec{k}_{1}-\vec{k}_{2}-\vec{k}_{3}, \vec{k}_{3}\right), \\
& \mathcal{G}_{2}\left(\vec{k}_{1}, \vec{k}_{2}, \vec{k}_{3}\right)=\mathcal{G}_{2}\left(\vec{k}_{1}, \vec{k}_{2},-\vec{k}_{1}-\vec{k}_{2}-\vec{k}_{3}\right) .
\end{aligned}
$$

The function $\mathcal{G}_{3}$ is symmetric in its first two arguments, and obeys the identity

$$
\mathcal{G}_{3}\left(\vec{k}_{1}, \vec{k}_{2}, \vec{k}_{3}\right)=\mathcal{G}_{3}\left(\vec{k}_{1}, \vec{k}_{2},-\vec{k}_{1}-\vec{k}_{2}-\vec{k}_{3}\right) .
$$

In addition taking the complex conjugate of Eq. (3.3c) gives the identity

$$
\mathcal{G}_{3}\left(\vec{k}_{1}, \vec{k}_{2}, \vec{k}_{3}\right)^{*}=\mathcal{G}_{3}\left(\vec{k}_{1}+\vec{k}_{2}+\vec{k}_{3},-\vec{k}_{3},-\vec{k}_{2}\right) .
$$

We also decompose the four point function into its disconnected or Gaussian piece, and its connected piece:

$$
\begin{aligned}
\mathcal{G}_{1}\left(\vec{k}_{1}, \vec{k}_{2}, \vec{k}_{3}\right)= & \delta\left(\vec{k}_{1}+\vec{k}_{2}\right) \mathcal{F}_{1}\left(\vec{k}_{1}\right) \mathcal{F}_{1}\left(\vec{k}_{3}\right)+\delta\left(\vec{k}_{1}+\vec{k}_{3}\right) \mathcal{F}_{1}\left(\vec{k}_{1}\right) \mathcal{F}_{1}\left(\vec{k}_{2}\right)+\delta\left(\vec{k}_{1}+\vec{k}_{4}\right) \mathcal{F}_{1}\left(\vec{k}_{1}\right) \mathcal{F}_{1}\left(\vec{k}_{2}\right) \\
& +\Gamma_{1}\left(\vec{k}_{1}, \vec{k}_{2}, \vec{k}_{3}\right) . \\
\mathcal{G}_{2}\left(\vec{k}_{1}, \vec{k}_{2}, \vec{k}_{3}\right)= & \delta\left(\vec{k}_{1}+\vec{k}_{2}\right) \mathcal{F}_{2}\left(\vec{k}_{1}\right) \mathcal{F}_{1}\left(\vec{k}_{3}\right)+\delta\left(\vec{k}_{1}+\vec{k}_{3}\right) \mathcal{F}_{2}\left(\vec{k}_{1}\right) \mathcal{F}_{1}\left(\vec{k}_{2}\right)+\delta\left(\vec{k}_{1}+\vec{k}_{4}\right) \mathcal{F}_{2}\left(\vec{k}_{1}\right) \mathcal{F}_{1}\left(\vec{k}_{2}\right) \\
& +\Gamma_{2}\left(\vec{k}_{1}, \vec{k}_{2}, \vec{k}_{3}\right) .
\end{aligned}
$$




$$
\begin{aligned}
\mathcal{G}_{3}\left(\vec{k}_{1}, \vec{k}_{2}, \vec{k}_{3}\right)= & \delta\left(\vec{k}_{1}+\vec{k}_{2}\right) \mathcal{F}_{1}\left(\vec{k}_{1}\right)^{*} \mathcal{F}_{1}\left(\vec{k}_{3}\right)+\delta\left(\vec{k}_{1}+\vec{k}_{3}\right) \mathcal{F}_{2}\left(\vec{k}_{1}\right) \mathcal{F}_{2}\left(\vec{k}_{2}\right)+\delta\left(\vec{k}_{1}+\vec{k}_{4}\right) \mathcal{F}_{2}\left(\vec{k}_{1}\right) \mathcal{F}_{2}\left(\vec{k}_{2}\right) \\
& +\Gamma_{3}\left(\vec{k}_{1}, \vec{k}_{2}, \vec{k}_{3}\right),
\end{aligned}
$$

Here the functions $\Gamma_{1}, \Gamma_{2}$ and $\Gamma_{2}$ parameterize the connected piece.

We note that Agullo and Parker [25] chose an initial density matrix which is diagonal on the eigenbasis of mode occupation number $\hat{N}_{\vec{k}}=\hat{A}_{\vec{k}}^{\dagger} \hat{A}_{\vec{k}}$ associated with the Bunch-Davies vacuum. This choice imposes the following restrictions on the functions $\mathcal{F}_{i}, \mathcal{H}_{i}$, and $\mathcal{G}_{i}$ :

$$
\begin{aligned}
\mathcal{F}_{1} & =0, \\
\mathcal{H}_{1} & =\mathcal{H}_{2}=0, \\
\mathcal{G}_{1} & =\mathcal{G}_{2}=0 .
\end{aligned}
$$

Our results for the spectrum and bispectrum below differ from theirs by terms involving these functions which vanish for their initial state.

Now there are two different versions of the Bunch-Davies initial vacuum state. There is the state $|0\rangle$ which satisfies $\hat{A}_{\vec{k}}|0\rangle=0$, the ground state of the free theory defined by the quadratic action (2.7). There is also the dressed vacuum $|\Omega\rangle$, defined as the ground state of the Hamiltonian of the full theory including nonlinear interaction terms given in Eq. (2.17) above. The standard Bunch-Davies result for the bispectrum, first calculated by Maldacena [52], is the result for the dressed vacuum. The functions $\mathcal{F}_{i}, \mathcal{H}_{2}, \mathcal{G}_{i}$ and $\Gamma_{i}$ all vanish in both vacua, $|0\rangle$ and $|\Omega\rangle$, to leading order in the interaction (2.17) (see Appendix A). However the function $\mathcal{H}_{1}$ vanishes only in the unperturbed vacuum $|0\rangle$, and not in the dressed vacuum $|\Omega\rangle$. We therefore define a modified version of this function by subtracting the dressed vacuum contribution

$$
\hat{\mathcal{H}}_{1} \equiv \mathcal{H}_{1}-\mathcal{H}_{1}^{0, \mathrm{dr}} \text {. }
$$

Here $\mathcal{H}_{1}^{0, \mathrm{dr}}$ means the value of $\mathcal{H}_{1}$ in the dressed vacuum $|\Omega\rangle$, which we compute in Appendix A. We will express our result below for the bispectrum in terms of $\mathcal{F}_{i}, \hat{\mathcal{H}}_{1}, \mathcal{H}_{2}$, and $\Gamma_{i}$, so that when these functions vanish our result reduces to that of Maldacena [52].

\subsection{Constraints from backreaction considerations}

The energy density and pressure due to the non-vacuum initial state must be small enough that it does not significantly perturb the inflationary background solution. We now review the order of magnitude estimate of this constraint [26, 29, 33]. As long as we are considering modes which are well inside the horizon, the stress energy density associated with the nonvacuum state consists of an energy density $\rho_{r}$ and a pressure $p_{r}=\rho_{r} / 3$. These must be small compared to the background energy density $\sim M_{p}^{2} H^{2}$ during inflation, yielding

$$
\rho_{r} \ll M_{p}^{2} H^{2} .
$$

A more precise restriction on the initial state can be obtained from the requirement that the slow roll parameters of the background expansion are not perturbed to be larger than indicated by observations. From the Friedmann equations we have

$$
\dot{H}=-\epsilon H^{2}=\frac{-1}{2 M_{p}^{2}}(p+\rho), \quad \ddot{H}=2 \epsilon \eta H^{3}=\frac{-1}{2 M_{p}^{2}}[\dot{p}-3 H(p+\rho)] .
$$

The pressure and density here consist of the usual contributions from the background inflaton field, together with the contributions $\rho_{r}$ and $p_{r}$ from the nonvacuum state of the perturbations. 
The changes in the slow roll parameters due to the radiation are then

$$
\Delta \epsilon \sim \rho_{r} /\left(M_{p}^{2} H^{2}\right), \quad \Delta \eta \sim \rho_{r} /\left(\epsilon M_{p}^{2} H^{2}\right) .
$$

Imposing that $\Delta \epsilon \lesssim \epsilon$ and $\Delta \eta \lesssim \eta$ yields the constraints [29]

$$
\begin{aligned}
\rho_{r} & \lesssim \epsilon M_{p}^{2} H^{2}, \\
\rho_{r} & \lesssim \epsilon M_{p}^{2} H^{2} .
\end{aligned}
$$

The constraints (3.14) are more precise than Eq. (3.11), and strongly restrict the number of quanta present in the initial state. Note that the upper bound (3.14a) is just the kinetic energy $\dot{\phi}^{2} \sim \epsilon M_{p}^{2} H^{2}$ of the inflaton field.

We impose the constraints (3.14) at the initial time $\tau_{0}$. If they are satisfied then, they will be satisfied at all subsequent times since the radiation energy density will fall off rapidly while the inflaton energy density is approximately constant.

We can approximately characterize initial states in terms of the mean mode occupation number $N_{\text {occ }}(k)$, related to the functions $\mathcal{F}_{1}$ and $\mathcal{F}_{2}$ by ${ }^{2}$

$$
N_{\mathrm{occ}} \sim \mathcal{F}_{1} \sim \mathcal{F}_{2}
$$

The energy density per logarithmic wavenumber is $\sim N_{\text {occ }}(k)(k / a)^{4}$. In order to explore the consequences of the constraints (3.14), we specialize to a class of states for which the mean occupation is a power law in some interval $k_{\min } \leq k \leq k_{\max }$ of comoving wavenumber,

$$
N_{\text {occ }}(k) \sim N_{0}\left(\frac{k}{k_{\min }}\right)^{n-4}
$$

and vanishes outside that interval. The corresponding energy density is $\rho_{r} \sim N_{0} E_{\max }^{4} \chi^{-4} f_{n}(\chi)$, where $E_{\max }=k_{\max } / a\left(\tau_{0}\right)$ is the maximum physical energy of occupied modes, $\chi=k_{\max } / k_{\min }$, and

$$
f_{n}(\chi)= \begin{cases}\ln \chi & n=0 \\ \frac{\chi^{n}-1}{n} & n \neq 0 .\end{cases}
$$

Combining these estimates with the constraint (3.14b) yields an upper bound on the mode occupation number

$$
N_{0} \lesssim \frac{\epsilon \eta M_{p}^{2} H^{2} \chi^{4}}{f_{n}(\chi) E_{\max }^{4}}
$$

We now explore the consequences of this bound under two different sets of assumptions, a robust and conservative set of assumptions in the next subsection, and a more speculative set in the following subsection.

\subsubsection{Constraints under conservative assumptions}

Our conservative assumptions are:

- At the initial time, the standard inflationary action (2.1) that we have assumed is valid as an effective field theory up to some cutoff energy scale $\Lambda$.

\footnotetext{
${ }^{2}$ For the special case of generalized vacuum states, the estimate (3.15) is valid for $N_{\text {occ }} \gtrsim 1$, but must be replaced by $\mathcal{F}_{2} \sim N_{\text {occ }}, \mathcal{F}_{1} \sim \sqrt{N_{\text {occ }}}$ when $N_{\text {occ }} \ll 1$, cf. Eq. (6.21) below. Because we are interested in general states we will use the estimate (3.15) in the remainder of this paper.
} 
- At the initial time, the occupied modes are all inside the horizon and below the cutoff, that is,

$$
H \leq k_{\min } / a \leq k_{\max } / a \leq \Lambda .
$$

This assumption is necessary for the validity of our method of computation of the backreaction due to the occupied modes.

It follows from these assumptions that $E_{\max } \geq \chi H$, where $\chi=k_{\max } / k_{\min }$. Combining this with the upper bound (3.18) and eliminating $\epsilon$ in favor of the measured amplitude of the power spectrum $\Delta_{\mathcal{R}}$ using Eq. (4.5) gives

$$
N_{0} \lesssim \frac{1}{f_{n}(\chi)} \frac{\eta}{8 \pi^{2} \Delta_{\mathcal{R}}^{2}}
$$

Now the observed value of the scalar spectral index, $n_{s}=1+2 \eta-4 \epsilon \sim 0.96$ [53], suggests that $\epsilon \sim \eta \sim 0.01$, absent any fine tuning. Also the observed value of $\Delta_{\mathcal{R}}$ is $\Delta_{\mathcal{R}} \sim 3 \times 10^{-5}$ [3]. Inserting these estimates finally gives the upper bound

$$
N_{0} \lesssim \frac{10^{5}}{f_{n}(\chi)}
$$

The final upper bound (3.21) on the mode occupation number depends strongly on the width $\chi=k_{\max } / k_{\min }$ of the band of occupied modes, as well as the power law index $n$. If only a small set of modes is occupied, $\chi \sim 1$, then the mode occupation number can be large compared to unity. However, if the entire range of modes visible in the CMB are occupied, so that $\chi \gtrsim 10^{3}$, then the constraint depends on the power law index. For example, for $n=0$, a scale invariant spectrum, $N_{0}$ can be large. For $n=4$ which corresponds to $N_{\text {occ }}(k) \sim$ (constant), we obtain the constraint that $N_{0} \lesssim 10^{-7}$, which is quite restrictive.

Note that $N_{0}$ is the value of $N_{\text {occ }}(k)$ at the minimum value of $k, k=k_{\min }$, and is also the largest value of $N_{\text {occ }}(k)$ as long as $n \leq 4$. Therefore the constraint (3.21) is an absolute upper bound for $N_{\text {occ }}(k)$ for $n \leq 4$. It will also be useful later to have constraints on the mode occupation number evaluated at the largest value of $k$,

$$
N_{1} \equiv N_{\text {occ }}\left(k_{\max }\right)=N_{0} \chi^{n-4},
$$

even though $N_{\text {occ }}(k)$ can exceed $N_{1}$. From Eq. (3.21) we find

$$
N_{1} \lesssim \frac{10^{5}}{\chi^{4-n} f_{n}(\chi)}=\frac{10^{5}}{\chi^{4}}\left[\frac{n}{1-\chi^{-n}}\right]
$$

The factor in square brackets is either of order unity or small, for $|n| \lesssim$ (a few), so we obtain $N_{1} \lesssim 10^{5} / \chi^{4}$. In particular $N_{1} \ll 1$ if the entire range of modes visible in the CMB are occupied, $\chi \gtrsim 10^{3}$.

Another interesting quantity to constrain is the maximum bandwidth $\chi_{\text {occ }}$ over which the occupation number exceeds unity. In other words, if $N_{\text {occ }} \geq 1$ for some range $k_{1} \leq k \leq k_{2}$ of values of $k$, what is the largest possible value of $\chi_{\mathrm{occ}}=k_{2} / k_{1}$ that is compatible with the backreaction constraint? We now argue that this maximum bandwidth is $\sim 25$.

Consider first the case $n<4$, in which the maximum value of $N_{\text {occ }}(k)$ is $N_{0}$ and is achieved at $k=k_{\min }$. Then if $N_{0}<1$, the occupation number never exceeds unity, and we set $\chi_{\mathrm{occ}}=1$ in this case (zero bandwidth). If $N_{0}>1$ the bandwidth is given by, from Eq. 
(3.16) $1=N_{0} \chi_{\mathrm{occ}}^{n-4}$, and combining these gives $\chi_{\mathrm{occ}}=\max \left(1, N_{0}^{1 /(4-n)}\right)$. However clearly $\chi_{\mathrm{occ}}$ cannot exceed $\chi=k_{\max } / k_{\min }$, the bandwidth in which we have assumed $N_{\text {occ }}$ is nonzero, so we obtain

$$
\chi_{\mathrm{occ}}=\min \left\{\chi, \max \left(1, N_{0}^{1 /(4-n)}\right)\right\} .
$$

A similar analysis for $n>4$ yields

$$
\chi_{\text {occ }}=\min \left\{\chi, \max \left(1, \chi N_{0}^{1 /(n-4)}\right)\right\} .
$$

Combining these results and using the upper bound (3.20) for $N_{0}$ gives

$$
\chi_{\text {occ }} \leq \min \left\{\chi, \max \left[1, \chi^{\Theta(n-4)}\left(\frac{G n}{\chi^{n}-1}\right)^{1 /|n-4|}\right]\right\},
$$

where $\Theta$ is the step function and $G=\eta /\left(8 \pi^{2} \Delta_{\mathcal{R}}^{2}\right) \sim 10^{5}$. We now maximize over all values of $n$ and over values of $\chi \geq 1$. The function of $n$ and $\chi$ on the right hand side of Eq. (3.26) is maximized at $n=4$ and at $\chi=(1+4 G)^{1 / 4}$, and the maximum value is $(1+4 G)^{1 / 4}$. Thus we obtain

$$
\chi_{\text {occ }} \lesssim(1+4 G)^{1 / 4} \sim 25 .
$$

Finally we note that the upper limit (3.27) on the bandwidth of occupied modes is weakened if one uses the less stringent backreaction constraint (3.14a) instead of the more stringent constraint (3.14b). This yields

$$
\chi_{\mathrm{occ}} \lesssim(1+4 G / \eta)^{1 / 4} \sim 100,
$$

which was the upper limit obtained in Ref. [54].

\subsubsection{Constraints under more speculative assumptions}

Next, an upper bound on $N_{0}$ which is somewhat stronger than the bound (3.21) can be obtained if one assumes that the backreaction is small not just at the chosen initial time, but also at all previous times, continuing into the past until the energies of the occupied modes are blueshifted up to the cutoff scale $\Lambda$. This assumption is not particularly well motivated, since it is possible, for example, for a multifield model to behave like a single field model in certain regimes, and going backwards in time a transition from single field behavior to to multifield behavior can occur while $k_{\max } / a(\tau) \ll \Lambda$. Nevertheless, if one makes this assumption, then from Eq. (3.18) evaluated at $E_{\max } \sim \Lambda$ we obtain

$$
N_{0} \lesssim \frac{\epsilon \eta M_{p}^{2} H^{2} \chi^{4}}{f_{n}(\chi) \Lambda^{4}}
$$

Next, we demand that the background inflationary solution be within the domain of the effective field theory. The action (2.1) will have correction terms such as $(\nabla \phi)^{4} / \Lambda^{4}$ that are suppressed by powers of the cutoff. This correction term must be small compared to $(\nabla \phi)^{2}$ when evaluated on the background solution, which yields the condition $\epsilon M_{p}^{2} H^{2} \ll \Lambda^{4}$. Combining this with Eq. (3.29) yields

$$
N_{0} \leq \eta \frac{\chi^{4}}{f_{n}(\chi)}=\left\{\begin{array}{lc}
\frac{\eta \chi^{4}}{\ln \chi} & n=0, \\
\frac{\eta n \chi^{4}}{\chi^{n}-1} & n \neq 0 .
\end{array}\right.
$$

Thus the mode occupation number must be small compared to unity if $\chi \sim 1$, assuming $\eta \sim 0.01$. It can be large however for $\chi \gg 1$ if $n<4$. 


\section{Scalar power spectrum}

The scalar power spectrum $\mathcal{P}_{\mathcal{R}}$ of the perturbations is is defined in terms of the momentum space equal time two-point function of the scalar curvature perturbation $\hat{\mathcal{R}}_{\vec{k}}(\tau)$ :

$$
\left\langle\hat{\mathcal{R}}_{\vec{k}_{1}}(\tau) \hat{\mathcal{R}}_{\vec{k}_{2}}(\tau)\right\rangle=(2 \pi)^{3} \delta\left(\vec{k}_{1}+\vec{k}_{2}\right) \mathcal{P}_{\mathcal{R}}\left(\vec{k}_{1}\right) .
$$

Here it is assumed that the conformal time $\tau$ is chosen to be any time after the modes have exited the horizon, so that the right hand side is independent of $\tau$. The dimensionless power spectrum $\Delta_{\mathcal{R}}$ is then defined by

$$
\mathcal{P}_{\mathcal{R}}(\vec{k}) \equiv \frac{2 \pi^{2}}{k^{3}} \Delta_{\mathcal{R}}^{2}(\vec{k})
$$

The scalar power spectrum has two contributions,

$$
\mathcal{P}_{\mathcal{R}}(\vec{k})=\mathcal{P}_{\mathcal{R}}^{(0)}(\vec{k})+\mathcal{P}_{\mathcal{R}}^{(1)}(\vec{k}),
$$

a leading order term $\mathcal{P}_{\mathcal{R}}^{(0)}(\vec{k})$ neglecting the effect of interactions, and a subleading term $\mathcal{P}_{\mathcal{R}}^{(1)}(\vec{k})$ arising from the interactions, cf. Sec. 2.2 above. The subleading term is smaller by a factor $\sim H \sqrt{\epsilon} / M_{p}$. We will consider both contributions.

\subsection{Leading order power spectrum}

For the Bunch-Davies vacuum initial state, this power spectrum is given by [3]

$$
\mathcal{P}_{\mathcal{R}, 0}^{(0)}(k)=\left|\mathcal{R}\left(k, \tau_{k}\right)\right|^{2},
$$

where the subscript 0 is used to indicate that this quantity is calculated in the Bunch-Davies vacuum, $\mathcal{R}(k, \tau)$ is the mode function defined by the condition (2.14), and $\tau_{k}$ is the time of horizon crossing given by $k=a\left(\tau_{k}\right) H$. Thus

$$
\Delta_{\mathcal{R}, 0}^{2}(k)=\frac{1}{2 M_{p}^{2} \epsilon\left(\tau_{k}\right)}\left[\frac{H\left(\tau_{k}\right)}{2 \pi}\right]^{2}
$$

Henceforth we shall denote the mode functions by $\mathcal{R}(k)$ only and restore the time dependence only when necessary.

For a general homogeneous initial state, we compute the leading order power spectrum by inserting the mode expansion (2.10) into the power spectrum definition (4.1), and simplifying using the definitions (3.1) of the two point functions $\mathcal{F}_{1}$ and $\mathcal{F}_{2}$. This yields ${ }^{3}$ (see, e.g., Ref. [55])

$$
\mathcal{P}_{\mathcal{R}}^{(0)}(\vec{k})=\mathcal{P}_{\mathcal{R}, 0}^{(0)}(\vec{k})\left[1+\mathcal{F}_{2}(-\vec{k})+\mathcal{F}_{2}(\vec{k})-2 \operatorname{Re}\left\{\mathcal{F}_{1}(\vec{k})\right\}\right],
$$

where Re means the real part of. Here we used the fact that $\mathcal{R}(k, \tau)$ is purely imaginary at late times according to Eq. (2.14). The terms in the square brackets after the 1 indicate the effect of the presence of quanta in the initial state. The result (4.6) agrees with that of Agullo

\footnotetext{
${ }^{3}$ An additional contribution to the change in the measured spectral index is the backreaction effect (3.13). Observations constrain the sum of the backreaction contribution and the direct contribution (4.6). One could imagine evading the backreaction constraints (3.14) via a cancellation between these two contributions, but this would require a fine tuning.
} 
and Parker [25], except that they omit the final term in the square bracket, which vanishes for their class of initial states, cf. Eq. (3.9a) above.

The leading order scalar power spectrum (4.6) has been calculated for variety of different choices of initial states $[23,25,36,38]$. For some specific initial states the deviation from the Bunch-Davies vacuum case vanishes [36, 38]. Current observational constraints on the flatness of the power spectrum constrain the initial state, ruling out models where the scale invariance of the power spectrum is strongly violated, for example Ref. [23].

\subsection{Subleading contributions to power spectrum due to interactions}

To calculate the power spectrum (4.1) to subleading order we use time-dependent perturbation theory. We choose the origin of conformal time so that the value of $\tau$ on the left hand side of Eq. (4.1) is $\tau=0$, that is, $\tau=0$ occurs after the modes exit the horizon. At leading order in perturbation theory we have

$$
\left\langle\hat{\mathcal{R}}_{\vec{k}_{1}}(0) \hat{\mathcal{R}}_{\vec{k}_{2}}(0)\right\rangle=\left\langle\hat{\mathcal{R}}_{\vec{k}_{1}}^{I}(0) \hat{\mathcal{R}}_{\vec{k}_{2}}^{I}(0)\right\rangle-i \int_{\tau_{0}}^{0} d \tau\left\langle\left[\hat{\mathcal{R}}_{\vec{k}_{1}}^{I}(0) \hat{\mathcal{R}}_{\vec{k}_{2}}^{I}(0), \hat{H}_{\mathrm{int}}^{I}(\tau)\right]\right\rangle+O\left(\hat{H}_{\mathrm{int}}^{2}\right) .
$$

Here the superscripts $I$ denote interaction picture operators, defined with respect to the initial conformal time $\tau_{0}$ at which we specify the initial state, and $\hat{H}_{\text {int }}$ is the interaction Hamiltonian given by Eq. (2.17). We now rewrite the field operators $\hat{\mathcal{R}}$ on the right hand side in terms of the redefined field operator $\hat{\mathcal{R}}_{c}$ using Eq. (2.15). We then insert the mode expansion (2.18) for the redefined interaction picture operators together with the explicit expression (2.17) for the interaction Hamiltonian, and simplify using the notations introduced in Sec. 3.1. We neglect any evolution in the slow roll parameters that occurs between the horizon exits of the different modes, that is, we treat these slow roll parameters as constants. The calculation is analogous to the calculation of the bispectrum detailed in Appendix B.

The final result for the subleading power spectrum is

$$
\mathcal{P}_{\mathcal{R}}^{(1)}(\vec{k})=-\frac{i H^{3}\left(\frac{3}{2} \epsilon-\eta\right)}{16 M_{p}^{3} k^{3} \epsilon^{\frac{3}{2}}} \Pi_{1}(\vec{k})-\frac{i H^{3}}{16 M_{p}^{3} k^{3} \epsilon^{\frac{1}{2}}} \Pi_{2}(\vec{k})+\frac{H^{3}}{8 \epsilon^{\frac{1}{2}} M_{p}^{3} k^{3}} \Pi_{3}(\vec{k}) .
$$

Here the functions $\Pi_{1}, \Pi_{2}$ and $\Pi_{3}$ are dimensionless functions of momentum. The function $\Pi_{1}$ is given by

$$
\begin{aligned}
\Pi_{1}(\vec{k})= & \tilde{\Phi}_{1}(\vec{k})-\tilde{\Phi}_{2,1}(\vec{k})-\tilde{\Phi}_{2,2}(\vec{k})-\tilde{\Phi}_{2,3}(\vec{k})+\tilde{\Phi}_{2,3}(-\vec{k})^{*}+\tilde{\Phi}_{2,2}(-\vec{k})^{*} \\
& +\tilde{\Phi}_{2,1}(-\vec{k})^{*}-\tilde{\Phi}_{1}(-\vec{k})^{*}+(\vec{k} \rightarrow-\vec{k})
\end{aligned}
$$

where

$$
\begin{aligned}
& \tilde{\Phi}_{1}(\vec{k})=\int d^{3} p p^{-3 / 2} q^{-3 / 2} k^{3 / 2} \hat{\mathcal{H}}_{1}(\vec{k}, \vec{p}), \\
& \tilde{\Phi}_{2,1}(\vec{k})=\int d^{3} p p^{-3 / 2} q^{-3 / 2} k^{3 / 2} \mathcal{H}_{2}(\vec{k}, \vec{p}), \\
& \tilde{\Phi}_{2,2}(\vec{k})=\int d^{3} p p^{-3 / 2} q^{-3 / 2} k^{3 / 2} \mathcal{H}_{2}(\vec{k}, \vec{q}), \\
& \tilde{\Phi}_{2,3}(\vec{k})=\int d^{3} p p^{-3 / 2} q^{-3 / 2} k^{3 / 2} \mathcal{H}_{2}(\vec{p}, \vec{q}),
\end{aligned}
$$


and $\vec{q}=-\vec{k}-\vec{p}$. Similarly the function $\Pi_{2}$ is given by

$$
\begin{aligned}
\Pi_{2}(\vec{k})= & \bar{\Phi}_{1}(\vec{k})-\bar{\Phi}_{2,1}(\vec{k})-\bar{\Phi}_{2,2}(\vec{k})-\bar{\Phi}_{2,3}(\vec{k})+\bar{\Phi}_{2,3}(-\vec{k})^{*}+\bar{\Phi}_{2,2}(-\vec{k})^{*} \\
& +\bar{\Phi}_{2,1}\left(-\vec{k}_{1}\right)^{*}-\bar{\Phi}_{1}\left(-\vec{k}_{1}\right)^{*}+(\vec{k} \rightarrow-\vec{k}),
\end{aligned}
$$

where the functions $\bar{\Phi}$ are defined by equations analogous to Eqs. (4.10) - (4.13) but with the factor $p^{-3 / 2} q^{-3 / 2} k^{3 / 2}$ in the integrand replaced with $p^{-3 / 2} q^{1 / 2} k^{-1 / 2}$. Lastly, the function $\Pi_{3}$ in Eq. (4.8) is given by

$$
\begin{aligned}
\Pi_{3}(\vec{k})= & \int d^{3} p \sqrt{p s k}\left[\frac{1}{k^{2}}+\frac{1}{p^{2}}+\frac{1}{s^{2}}\right] \\
& \times\left\{h(p, s)\left[\hat{\mathcal{H}}_{1}(\vec{p}, \vec{s})-\mathcal{H}_{2}(\vec{p}, \vec{s})\right]-h(-p, s)\left[\mathcal{H}_{2}(-\vec{k}, \vec{p})-\mathcal{H}_{2}(-\vec{s}, \vec{k})^{*}\right]\right. \\
& \left.-h(p,-s)\left[\mathcal{H}_{2}(-\vec{k},-\vec{s})-\mathcal{H}_{2}(-\vec{p}, \vec{k})^{*}\right]+h(-p,-s)\left[\mathcal{H}_{2}(-\vec{p},-\vec{s})^{*}-\hat{\mathcal{H}}_{1}(-\vec{p}, \vec{k})^{*}\right]\right\} \\
& +(\vec{k} \rightarrow-\vec{k}),
\end{aligned}
$$

where $\vec{s}=\vec{k}-\vec{p}$. Here the function $h(a, b)$ is

$$
\begin{aligned}
h(a, b) & =\int_{0}^{\tau_{0}} d \tau\left[e^{-i \tau(a+b+k)}-e^{-i \tau(a+b-k)}\right] \\
& =\frac{i}{a+b-k}\left[1-e^{-i \tau_{0}(a+b-k)}\right]-\frac{i}{a+b+k}\left[1-e^{-i \tau_{0}(a+b+k)}\right] .
\end{aligned}
$$

We note that the subleading contribution (4.8) to the power spectrum depends only on the three point function of the initial state, parameterized by the functions $\mathcal{H}_{i}$, and so it vanishes for Gaussian initial states. This includes the Bunch-Davies vacuum state.

\section{Primordial non-Gaussianities: bispectrum of scalar perturbations}

To predict the non-Gaussianity of the primordial fluctuations one needs to consider higher order correlation functions. The full computation of the momentum space three point correlation function of the comoving curvature perturbation field for a vacuum initial state in a single field model was done by Maldacena [52]. Here we calculate the three point function for the class of non-vacuum homogeneous initial states described in section 3. This calculation is a generalization of the one done by Agullo and Parker [25].

The three point function of the comoving curvature perturbation field $\hat{\mathcal{R}}_{\vec{k}}(\tau)$ is parameterized as

$$
\left\langle\hat{\mathcal{R}}_{\vec{k}_{1}}(\tau) \hat{\mathcal{R}}_{\vec{k}_{2}}(\tau) \hat{\mathcal{R}}_{\vec{k}_{3}}(\tau)\right\rangle=(2 \pi)^{3} \delta\left(\vec{k}_{1}+\vec{k}_{2}+\vec{k}_{3}\right) \mathcal{B}\left(\vec{k}_{1}, \vec{k}_{2}, \vec{k}_{3}\right)
$$

where $\mathcal{B}\left(\vec{k}_{1}, \vec{k}_{2}, \vec{k}_{3}\right)$ is called the bispectrum. Here it is assumed that the conformal time $\tau$ is chosen to be any time after all three modes have exited the horizon, so that the right hand side is independent of $\tau$. We now fix values of $\vec{k}_{1}, \vec{k}_{2}, \vec{k}_{3}$, which we assume to be nonzero, and we choose the origin of conformal time as before so that the value of $\tau$ on the left hand side of Eq. (5.1) is $\tau=0$, that is, $\tau=0$ occurs after the modes exit the horizon. 
To calculate the correlation function (5.1) we use time-dependent perturbation theory as in Sec. 4.2 above. At leading order in perturbation theory we have

$$
\begin{aligned}
\left\langle\hat{\mathcal{R}}_{\vec{k}_{1}}(0) \hat{\mathcal{R}}_{\vec{k}_{2}}(0) \hat{\mathcal{R}}_{\vec{k}_{3}}(0)\right\rangle= & \left\langle\hat{\mathcal{R}}_{\vec{k}_{1}}^{I}(0) \hat{\mathcal{R}}_{\vec{k}_{2}}^{I}(0) \hat{\mathcal{R}}_{\vec{k}_{3}}^{I}(0)\right\rangle \\
& -i \int_{\tau_{0}}^{0} d \tau\left\langle\left[\hat{\mathcal{R}}_{\vec{k}_{1}}^{I}(0) \hat{\mathcal{R}}_{\vec{k}_{2}}^{I}(0) \hat{\mathcal{R}}_{\vec{k}_{3}}^{I}(0), \hat{H}_{\mathrm{int}}^{I}(\tau)\right]\right\rangle+O\left(\hat{H}_{\mathrm{int}}^{2}\right) .
\end{aligned}
$$

Here the superscripts $I$ denote interaction picture operators, $\hat{H}_{\text {int }}$ is the interaction Hamiltonian given by Eq. (2.17), and $\tau_{0}$ is the conformal time at which we specify the initial state. We now rewrite the field operators $\hat{\mathcal{R}}$ on the right hand side in terms of the redefined field operator $\hat{\mathcal{R}}_{c}$ using Eq. (2.15). We then insert the mode expansion (2.18) for the redefined interaction picture operators together with the explicit expression (2.17) for the interaction Hamiltonian, and simplify using the notations introduced in Sec. 3.1. We neglect any evolution in the slow roll parameters that occurs between the horizon exits of the different modes, that is, we treat these slow roll parameters as constants. The details of this calculation are given in Appendix B.

We note that the interaction Hamiltonian (2.17) is cubic in the fields, so the second term in Eq. (5.2) contains six factors of field operators. However, these factors occur inside a commutator, and when this commutator is evaluated explicitly the number of factors of field operators is reduced to four. Therefore we only require the two, three and four point functions of the initial state, and not any higher point functions.

The final result for the bispectrum can be written as follows:

$$
\mathcal{B}\left(\vec{k}_{1}, \vec{k}_{2}, \vec{k}_{3}\right)=\mathcal{B}_{0, \mathrm{dr}}\left(\vec{k}_{1}, \vec{k}_{2}, \vec{k}_{3}\right)+\mathcal{B}_{I I}\left(\vec{k}_{1}, \vec{k}_{2}, \vec{k}_{3}\right)+\mathcal{B}_{I I I}\left(\vec{k}_{1}, \vec{k}_{2}, \vec{k}_{3}\right)+\mathcal{B}_{I V}\left(\vec{k}_{1}, \vec{k}_{2}, \vec{k}_{3}\right) .
$$

Here $\mathcal{B}_{0, \mathrm{dr}}$ is the bispectrum for the initial, dressed, Bunch-Davies vacuum, as calculated by Maldacena [52]. The remaining terms are corrections due to the non-vacuum initial state. The term $\mathcal{B}_{I I}$ is determined by the two point function of the initial state, $\mathcal{B}_{I I I}$ by the three point function, and $\mathcal{B}_{I V}$ by the connected part of the four point function. We now discuss these various contributions in turn.

\subsection{Vacuum contribution}

The vacuum contribution is [52]

$$
\mathcal{B}_{0, \mathrm{dr}}\left(\vec{k}_{1}, \vec{k}_{2}, \vec{k}_{3}\right)=\frac{H^{4}}{16 M_{p}^{4} \epsilon^{2} k_{1}^{3} k_{2}^{3} k_{3}^{3}}\left[\frac{1}{2} \epsilon \sigma_{1} \sigma_{2}-(\eta-\epsilon) \sigma_{3}+2 \epsilon\left(\sigma_{2}^{2}-\sigma_{4}\right) / \sigma_{1}\right],
$$

where we have defined

$$
\sigma_{p} \equiv \sum_{i=1}^{3} k_{i}^{p}
$$

for $p=1,2,3,4$. This is the result that applies when $\mathcal{F}_{i}=\hat{\mathcal{H}}_{1}=\mathcal{H}_{2}=\Gamma_{i}=0$.

\subsection{Contribution from two point function}

The piece $\mathcal{B}_{I I}$ of the bispectrum depends only on the functions $\mathcal{F}_{1}(\vec{k})$ and $\mathcal{F}_{2}(\vec{k})$ that parameterize the initial two point function, given by Eqs. (3.1) and (3.10) above. For a Gaussian 
initial state, as studied previously in Refs. [27-33, 36, 37], this gives the entire bispectrum. The result is

$$
\begin{aligned}
\mathcal{B}_{I I}\left(\vec{k}_{1}, \vec{k}_{2}, \vec{k}_{3}\right)= & \frac{H^{4}\left(\frac{3}{2} \epsilon-\eta\right)}{16 M_{p}^{4} \epsilon^{2} k_{1}^{2} k_{2}^{2} k_{3}^{2}} \mathcal{A}_{I I}\left(\vec{k}_{1}, \vec{k}_{2}, \vec{k}_{3}\right)+\frac{H^{4}}{32 M_{p}^{4} \epsilon k_{1}^{2} k_{2}^{2} k_{3}^{2}} \tilde{\mathcal{A}}_{I I}\left(\vec{k}_{1}, \vec{k}_{2}, \vec{k}_{3}\right) \\
& -\frac{i H^{4}}{8 M_{p}^{4} \epsilon k_{1}^{2} k_{2}^{2} k_{3}^{2}} \hat{\mathcal{A}}_{I I}\left(\vec{k}_{1}, \vec{k}_{2}, \vec{k}_{3}\right) .
\end{aligned}
$$

Here the dimensionless amplitude $\mathcal{A}_{I I}$ is given by

$$
\mathcal{A}_{I I}=\frac{k_{3}^{2}}{k_{1} k_{2}}\left[\overline{\mathcal{F}}\left(\vec{k}_{1}\right) \overline{\mathcal{F}}\left(\vec{k}_{2}\right)+\overline{\mathcal{F}}\left(\vec{k}_{1}\right)+\overline{\mathcal{F}}\left(\vec{k}_{2}\right)\right]+\text { cyclic perms }
$$

where "cyclic perms" means two terms obtained from the original term by cyclicly permuting $\vec{k}_{1}, \vec{k}_{2}$ and $\vec{k}_{3}$. Also we have defined the function

$$
\overline{\mathcal{F}}(\vec{k}) \equiv \mathcal{F}_{2}(\vec{k})+\mathcal{F}_{2}(-\vec{k})-2 \operatorname{Re} \mathcal{F}_{1}(\vec{k}),
$$

which is same combination of $\mathcal{F}_{1}$ and $\mathcal{F}_{2}$ that appears in the power spectrum (4.6). The dimensionless amplitude $\tilde{\mathcal{A}}_{I I}$ is given by

$$
\tilde{\mathcal{A}}_{I I}=\frac{k_{1}^{2}+k_{2}^{2}}{k_{1} k_{2}}\left[\overline{\mathcal{F}}\left(\vec{k}_{1}\right) \overline{\mathcal{F}}\left(\vec{k}_{2}\right)+\overline{\mathcal{F}}\left(\vec{k}_{1}\right)+\overline{\mathcal{F}}\left(\vec{k}_{2}\right)\right]+\text { cyclic perms }
$$

The dimensionless amplitude $\hat{\mathcal{A}}_{I I}$ is given by

$$
\begin{aligned}
\hat{\mathcal{A}}_{I I}= & k_{1} k_{2} k_{3} \sum_{i=1}^{3} \frac{1}{k_{i}^{2}}\left(r\left(-k_{t}, \tilde{k}_{3}\right)\left[\mathcal{F}_{2}\left(\vec{k}_{1}\right) \mathcal{F}_{2}\left(\vec{k}_{2}\right)+\mathcal{F}_{1}\left(\vec{k}_{1}\right) \mathcal{F}_{1}\left(\vec{k}_{2}\right)\right]\right. \\
& +\left\{r\left(-\tilde{k}_{2}, \tilde{k}_{1}\right)\left[\mathcal{F}_{2}\left(-\vec{k}_{1}\right) \mathcal{F}_{1}\left(\vec{k}_{2}\right)+\mathcal{F}_{2}\left(\vec{k}_{2}\right) \mathcal{F}_{1}^{*}\left(\vec{k}_{1}\right)\right]+r\left(-k_{t}, \tilde{k}_{3}\right) \mathcal{F}_{2}\left(\vec{k}_{2}\right) \mathcal{F}_{1}\left(\vec{k}_{1}\right)\right. \\
& \left.+r\left(\tilde{k}_{1},-\tilde{k}_{2}\right)\left[\mathcal{F}_{1}\left(\vec{k}_{2}\right) \mathcal{F}_{1}\left(\vec{k}_{1}\right)^{*}+\mathcal{F}_{2}\left(\vec{k}_{2}\right) \mathcal{F}_{2}\left(-\vec{k}_{1}\right)\right]+r\left(k_{t},-\tilde{k}_{3}\right) \mathcal{F}_{2}\left(-\vec{k}_{1}\right) \mathcal{F}_{1}\left(\vec{k}_{2}\right)^{*}+\left(\vec{k}_{1} \leftrightarrow \vec{k}_{2}\right)\right\} \\
& +r\left(-\tilde{k}_{3}, k_{t}\right)\left[\mathcal{F}_{2}\left(-\vec{k}_{1}\right) \mathcal{F}_{2}\left(-\vec{k}_{2}\right)+\mathcal{F}_{1}\left(\vec{k}_{1}\right)^{*} \mathcal{F}_{1}\left(\vec{k}_{2}\right)^{*}\right] \\
& \left.+r\left(-k_{t},-\tilde{k}_{3}\right) \mathcal{F}_{2}\left(\vec{k}_{3}\right)+r\left(\tilde{k}_{3}, k_{t}\right) \mathcal{F}_{2}\left(-\vec{k}_{3}\right)+r\left(-\tilde{k}_{3},-k_{t}\right) \mathcal{F}_{1}\left(\vec{k}_{3}\right)+r\left(k_{t}, \tilde{k}_{3}\right) \mathcal{F}_{1}\left(\vec{k}_{3}\right)^{*}\right) \\
& + \text { cyclic perms, }
\end{aligned}
$$

where

$$
k_{t} \equiv k_{1}+k_{2}+k_{3}, \quad \tilde{k}_{i} \equiv k_{t}-2 k_{i} .
$$

Here we have defined the function $r(a, b)$ by

$$
\begin{aligned}
r(a, b) & =\int_{\tau_{0}}^{0} d \tau\left[e^{i \tau a}-e^{i \tau b}\right] \\
& =\frac{1}{i a}\left[1-e^{i \tau_{0} a}\right]-\frac{1}{i b}\left[1-e^{i \tau_{0} b}\right] .
\end{aligned}
$$




\subsection{Contribution from three point function}

The piece $\mathcal{B}_{I I I}$ of the bispectrum is

$$
\mathcal{B}_{I I I}\left(\vec{k}_{1}, \vec{k}_{2}, \vec{k}_{3}\right)=-\frac{i H^{3}}{8 M_{p}^{3} \epsilon^{3 / 2} k_{1}^{2} k_{2}^{2} k_{3}^{2}} \mathcal{A}_{I I I}\left(\vec{k}_{1}, \vec{k}_{2}, \vec{k}_{3}\right)
$$

where the dimensionless amplitude $\mathcal{A}_{I I I}$ is given by

$$
\begin{aligned}
\mathcal{A}_{I I I}= & \left(k_{1} k_{2} k_{3}\right)^{1 / 2}\left[\hat{\mathcal{H}}_{1}\left(\vec{k}_{1}, \vec{k}_{2}\right)-\hat{\mathcal{H}}_{1}\left(-\vec{k}_{1},-\vec{k}_{2}\right)^{*}-\mathcal{H}_{2}\left(\vec{k}_{1}, \vec{k}_{2}\right)+\mathcal{H}_{2}\left(-\vec{k}_{1},-\vec{k}_{2}\right)^{*}-\mathcal{H}_{2}\left(\vec{k}_{1}, \vec{k}_{3}\right)\right. \\
& \left.+\mathcal{H}_{2}\left(-\vec{k}_{1},-\vec{k}_{3}\right)^{*}-\mathcal{H}_{2}\left(\vec{k}_{2}, \vec{k}_{3}\right)+\mathcal{H}_{2}\left(-\vec{k}_{2},-\vec{k}_{3}\right)^{*}\right],
\end{aligned}
$$

where the functions $\hat{\mathcal{H}}_{1}$ and $\mathcal{H}_{2}$ are defined in Eqs. (3.2) and (3.10) above.

\subsection{Contribution from connected piece of four point function}

This piece of the bispectrum is determined by the functions $\Gamma_{i}\left(\vec{k}_{1}, \vec{k}_{2}, \vec{k}_{3}\right)$ for $i=1,2,3$ that parameterize the connected piece of the four point function of the initial state, defined by Eqs. (3.3), (3.8) and (3.10) above. In order to give the result we first define some notations. We define the functions $\Theta_{i, j}\left(\vec{k}_{1}, \vec{k}_{2}\right)$ for $1 \leq i \leq 3$ and $1 \leq j \leq 4$ by

$$
\begin{aligned}
\Theta_{i, 1}\left(\vec{k}_{1}, \vec{k}_{2}\right) & =\int d^{3} p p^{-3 / 2} q^{1 / 2} \Gamma_{i}\left(\vec{k}_{1}, \vec{k}_{2}, \vec{p}\right), \\
\Theta_{i, 2}\left(\vec{k}_{1}, \vec{k}_{2}\right) & =\int d^{3} p p^{-3 / 2} q^{1 / 2} \Gamma_{i}\left(\vec{p}, \vec{k}_{1}, \vec{k}_{2}\right), \\
\Theta_{i, 3}\left(\vec{k}_{1}, \vec{k}_{2}\right) & =\int d^{3} p p^{-3 / 2} q^{1 / 2} \Gamma_{i}\left(\vec{q}, \vec{k}_{1}, \vec{k}_{2}\right), \\
\Theta_{i, 4}\left(\vec{k}_{1}, \vec{k}_{2}\right) & =\int d^{3} p p^{-3 / 2} q^{1 / 2} \Gamma_{i}\left(\vec{p}, \vec{q}, \vec{k}_{1}\right) .
\end{aligned}
$$

Here on the right hand sides $\vec{k}_{3}=-\vec{k}_{1}-\vec{k}_{2}$ and $\vec{q}=\vec{k}_{3}-\vec{p}$. We also define barred versions of some of these functions by modifying the weighting factor in the integrand:

$$
\begin{aligned}
& \bar{\Theta}_{i, 1}\left(\vec{k}_{1}, \vec{k}_{2}\right)=\int d^{3} p p^{-3 / 2} q^{-3 / 2} \Gamma_{i}\left(\vec{k}_{1}, \vec{k}_{2}, \vec{p}\right), \\
& \bar{\Theta}_{i, 2}\left(\vec{k}_{1}, \vec{k}_{2}\right)=\int d^{3} p p^{-3 / 2} q^{-3 / 2} \Gamma_{i}\left(\vec{p}, \vec{k}_{1}, \vec{k}_{2}\right), \\
& \bar{\Theta}_{i, 4}\left(\vec{k}_{1}, \vec{k}_{2}\right)=\int d^{3} p p^{-3 / 2} q^{-3 / 2} \Gamma_{i}\left(\vec{p}, \vec{q}, \vec{k}_{1}\right) .
\end{aligned}
$$

These functions are not all independent; it follows from the identities (3.4) - (3.6) that $\bar{\Theta}_{1,1}=\bar{\Theta}_{1,2}=\bar{\Theta}_{1,4}, \bar{\Theta}_{2,2}=\bar{\Theta}_{2,4} \Theta_{1,1}=\Theta_{1,2}=\Theta_{1,3}=\Theta_{1,4}$, and $\Theta_{2,2}=\Theta_{2,4}$. Also the functions $\bar{\Theta}_{1,1}, \bar{\Theta}_{2,2}, \bar{\Theta}_{3,1}, \bar{\Theta}_{3,4}, \Theta_{1,1}, \Theta_{2,2}, \Theta_{2,3}, \Theta_{3,1}$ and $\Theta_{3,4}$ are all symmetric under the interchange of $\vec{k}_{1}$ and $\vec{k}_{2}$.

The result for the bispectrum is

$$
\begin{aligned}
\mathcal{B}_{I V}\left(\vec{k}_{1}, \vec{k}_{2}, \vec{k}_{3}\right)= & \frac{H^{4}\left(\frac{3}{2} \epsilon-\eta\right)}{32 M_{p}^{4} \epsilon^{2} k_{1}^{2} k_{2}^{2} k_{3}^{2}} \mathcal{A}_{I V}\left(\vec{k}_{1}, \vec{k}_{2}, \vec{k}_{3}\right)+\frac{H^{4}}{32 M_{p}^{4} \epsilon k_{1}^{2} k_{2}^{2} k_{3}^{2}} \tilde{\mathcal{A}}_{I V}\left(\vec{k}_{1}, \vec{k}_{2}, \vec{k}_{3}\right) \\
& +\frac{i H^{4}}{16 M_{p}^{4} \epsilon k_{1}^{2} k_{2}^{2} k_{3}^{2}} \hat{\mathcal{A}}_{I V}\left(\vec{k}_{1}, \vec{k}_{2}, \vec{k}_{3}\right) .
\end{aligned}
$$


Here the dimensionless amplitude $\mathcal{A}_{I V}$ is given by

$$
\begin{aligned}
\mathcal{A}_{I V}= & k_{3}^{2} \sqrt{k_{1} k_{2}}\left[\bar{\Theta}_{1,1}\left(\vec{k}_{1}, \vec{k}_{2}\right)+\bar{\Theta}_{1,1}\left(-\vec{k}_{1},-\vec{k}_{2}\right)^{*}-2 \bar{\Theta}_{2,2}\left(\vec{k}_{1}, \vec{k}_{2}\right)-\bar{\Theta}_{2,1}\left(\vec{k}_{2}, \vec{k}_{1}\right)\right. \\
& -\bar{\Theta}_{2,1}\left(-\vec{k}_{1},-\vec{k}_{2}\right)^{*}-\bar{\Theta}_{2,1}\left(\vec{k}_{1}, \vec{k}_{2}\right)-\bar{\Theta}_{2,1}\left(-\vec{k}_{2},-\vec{k}_{1}\right)^{*}-2 \bar{\Theta}_{2,2}\left(-\vec{k}_{1},-\vec{k}_{2}\right)^{*}+\bar{\Theta}_{3,4}\left(\vec{k}_{1}, \vec{k}_{2}\right) \\
& \left.+2 \bar{\Theta}_{3,2}\left(\vec{k}_{2}, \vec{k}_{1}\right)+2 \bar{\Theta}_{3,2}\left(\vec{k}_{1}, \vec{k}_{2}\right)+\bar{\Theta}_{3,1}\left(\vec{k}_{1}, \vec{k}_{2}\right)\right] \quad+\text { cyclic perms. }
\end{aligned}
$$

The dimensionless amplitude $\tilde{\mathcal{A}}_{I V}$ is given by

$$
\begin{aligned}
\tilde{\mathcal{A}}_{I V}= & \sqrt{k_{1} k_{2}}\left[\Theta_{1,1}\left(\vec{k}_{1}, \vec{k}_{2}\right)+\Theta_{1,1}\left(-\vec{k}_{1},-\vec{k}_{2}\right)^{*}-\Theta_{2,2}\left(\vec{k}_{1}, \vec{k}_{2}\right)-\Theta_{2,3}\left(\vec{k}_{1}, \vec{k}_{2}\right)-\Theta_{2,1}\left(\vec{k}_{2}, \vec{k}_{1}\right)\right. \\
& -\Theta_{2,1}\left(-\vec{k}_{1},-\vec{k}_{2}\right)^{*}-\Theta_{2,1}\left(\vec{k}_{1}, \vec{k}_{2}\right)-\Theta_{2,1}\left(-\vec{k}_{2},-\vec{k}_{1}\right)^{*}-\Theta_{2,2}\left(-\vec{k}_{1},-\vec{k}_{2}\right)^{*} \\
& -\Theta_{2,3}\left(-\vec{k}_{1},-\vec{k}_{2}\right)^{*}+\Theta_{3,4}\left(\vec{k}_{1}, \vec{k}_{2}\right)+\Theta_{3,2}\left(\vec{k}_{2}, \vec{k}_{1}\right)+\Theta_{3,3}\left(\vec{k}_{2}, \vec{k}_{1}\right)+\Theta_{3,2}\left(\vec{k}_{1}, \vec{k}_{2}\right) \\
& \left.+\Theta_{3,3}\left(\vec{k}_{1}, \vec{k}_{2}\right)+\Theta_{3,1}\left(\vec{k}_{1}, \vec{k}_{2}\right)\right] \quad+\text { cyclic perms. }
\end{aligned}
$$

Finally the dimensionless amplitude $\hat{\mathcal{A}}_{I V}$ is given by

$$
\begin{aligned}
\hat{\mathcal{A}}_{I V}= & \sqrt{k_{1} k_{2}} k_{3} \int d^{3} p \sqrt{p q}\left[\frac{1}{k_{3}^{2}}+\frac{1}{p^{2}}+\frac{1}{q^{2}}\right]\left\{h(p, q)\left[\Gamma_{1}\left(\vec{k}_{1}, \vec{k}_{2}, \vec{p}\right)+\Gamma_{3}\left(\vec{k}_{1}, \vec{k}_{2}, \vec{p}\right)\right]\right. \\
& -h(p,-q)\left[\Gamma_{2}\left(\vec{q}, \vec{k}_{1}, \vec{k}_{2}\right)+\Gamma_{2}\left(-\vec{p},-\vec{k}_{1},-\vec{k}_{2}\right)^{*}\right]-h(-p, q)\left[\Gamma_{2}\left(\vec{p}, \vec{k}_{1}, \vec{k}_{2}\right)+\Gamma_{2}\left(-\vec{q},-\vec{k}_{1},-\vec{k}_{2}\right)^{*}\right] \\
& +h(-p,-q)\left[\Gamma_{3}\left(\vec{q}, \vec{p}, \vec{k}_{1}\right)+\Gamma_{1}\left(-\vec{p},-\vec{k}_{1},-\vec{k}_{2}\right)^{*}\right]-h(p, q)\left[\Gamma_{2}\left(\vec{k}_{1}, \vec{k}_{2}, \vec{p}\right)+\Gamma_{2}\left(\vec{k}_{2}, \vec{k}_{1}, \vec{p}\right)\right] \\
& -h(p,-q)\left[\Gamma_{3}\left(\vec{q}, \vec{k}_{1}, \vec{k}_{2}\right)+\Gamma_{3}\left(\vec{q}, \vec{k}_{2}, \vec{k}_{1}\right)\right]+h(-p, q)\left[\Gamma_{3}\left(\vec{p}, \vec{k}_{1}, \vec{k}_{2}\right)+\Gamma_{3}\left(\vec{p}, \vec{k}_{2}, \vec{k}_{1}\right)\right] \\
& \left.-h(-p,-q)\left[\Gamma_{2}\left(-\vec{k}_{1},-\vec{k}_{2},-\vec{p}\right)^{*}+\Gamma_{2}\left(-\vec{k}_{2},-\vec{k}_{1},-\vec{p}\right)^{*}\right]\right\} \\
& + \text { cyclic perms, }
\end{aligned}
$$

where as before $\vec{k}_{3}=-\vec{k}_{1}-\vec{k}_{2}$ and $\vec{q}=\vec{k}_{3}-\vec{p}$. Also we have defined the function $h(a, b)$ by

$$
\begin{aligned}
h(a, b) & =\int_{0}^{\tau_{0}} d \tau\left[e^{-i \tau\left(a+b+k_{3}\right)}-e^{-i \tau\left(a+b-k_{3}\right)}\right] \\
& =\frac{i}{a+b-k_{3}}\left[1-e^{-i \tau_{0}\left(a+b-k_{3}\right)}\right]-\frac{i}{a+b+k_{3}}\left[1-e^{-i \tau_{0}\left(a+b+k_{3}\right)}\right] .
\end{aligned}
$$

\section{Order of magnitude estimates and discussion}

We now discuss the implications of our results for the bispectrum by making some order of magnitude estimates. The results depends on a number of parameters and quantities:

- The ratio of the Hubble and Planck scales, $H / M_{p}$.

- The slow roll parameters $\epsilon$ and $\eta$. For our order of magnitude estimates we will assume that $\epsilon \sim \eta$. 
- In the isotropic case, the dependence on the wave vectors $\vec{k}_{1}, \vec{k}_{2}$ and $\vec{k}_{3}$ can be parameterized in terms of a dependence on the overall scale

$$
k \equiv\left(k_{1} k_{2} k_{3}\right)^{1 / 3}
$$

and the dependence on the two parameters $x_{2}=k_{2} / k_{1}$ and $x_{3}=k_{3} / k_{1}$ which characterizes the "shape" of the bispectrum.

- Some conventional terminology for the various limits in the space of shape parameters $x_{2}$ and $x_{3}$ is as follows. Conventionally one orders the momenta so that $x_{3}<x_{2}<1$, and the triangle inequality gives $x_{2}+x_{3}>1$. These inequalities restrict the allowed configurations to a triangle in the $\left(x_{2}, x_{3}\right)$ plane, see, for example, Fig. 30 of Ref. [3]. The three corners of the triangle are the squeezed limit $x_{3} \rightarrow 0, x_{2} \rightarrow 1$, the equilateral limit $x_{3} \rightarrow 1, x_{2} \rightarrow 1$, and the folded limit $x_{3} \rightarrow 1 / 2, x_{2} \rightarrow 1 / 2$. Two of the edges of the triangle are the elongated limit $x_{2}+x_{3} \rightarrow 1$ with $0 \leq x_{3} \leq 1 / 2$, and the isosceles limit $x_{2}-x_{3} \rightarrow 0$ with $1 / 2 \leq x_{3} \leq 1$.

- Suppose that $\mathcal{B}\left(k, x_{2}, x_{3}\right) / \mathcal{P}_{\mathcal{R}}(k)^{2}$ is proportional to a known function $\mathcal{S}\left(k, x_{2}, x_{3}\right)$, which is called as shape function. Then we can define a dimensionless parameter $f_{\mathrm{NL}}$ for that shape function, a measure of the amplitude of the bispectrum. The conventional definition is [3]

$$
\mathcal{B}\left(k, x_{2}, x_{3}\right)=\frac{18}{5} f_{\mathrm{NL}} \mathcal{P}_{\mathcal{R}}(k)^{2} \frac{\mathcal{S}\left(k, x_{2}, x_{3}\right)}{\mathcal{S}(k, 1,1)} .
$$

\subsection{Generic initial states}

Let us start off by neglecting the shape dependence, assuming that $k_{1} \sim k_{2} \sim k_{3} \sim k$. In this case the vacuum bispectrum (5.4) scales as $\mathcal{B}_{0, \mathrm{dr}} \sim H^{4} /\left(M_{p}^{4} \epsilon k^{6}\right)$. Suppose now that the occupation number of the initial state is of order unity. Suppose also that the initial state is "generic" in the sense that all the dimensionless amplitudes $\mathcal{A}$ defined in Sec. 5 are of order unity, so that, for example, the three point function is not suppressed compared to the two point function, etc. If we are agnostic about the origin of the non-Bunch-Davies initial state, then this genericity assumption is well motivated. However, specific scenarios for generating initial states can give rise to suppression of the three point function with respect to the two and four point functions, and violate our genericity assumption. For example, this occurs in the second class of secnarios discussed in the introduction, where the non Bunch-Davies initial states arise as a result of new physics at high energies which has been integrated out. See, for example, Ref. [56]. This important class of scenarios yields non-generic initial states. Nevertheless, for the remainder of this subsection we will restrict attention to generic initial states.

With the assumption that all the dimensionless amplitudes $\mathcal{A}$ are of order unity, we find that the results (5.6) and (5.17) for the contributions from the two and four point functions are of the same order as the vacuum contribution:

$$
\mathcal{B}_{I I} \sim \mathcal{B}_{I V} \sim \frac{H^{4}}{M_{p}^{4} \epsilon k^{6}} \sim \mathcal{B}_{0, \mathrm{dr}} .
$$

These contributions are therefore small and hard to detect. By contrast, the contribution (5.13) from the three point function scales as

$$
\mathcal{B}_{I I I} \sim \frac{H^{3}}{M_{p}^{3} \epsilon^{3 / 2} k^{6}} \sim \frac{M_{p}}{H \sqrt{\epsilon}} \mathcal{B}_{0, \mathrm{dr}} .
$$


We now eliminate $M_{p} / H$ in favor of $\epsilon$ and the power spectrum $\Delta_{\mathcal{R}}$ using Eq. (4.5). This gives

$$
\mathcal{B}_{I I I} \sim \frac{1}{\epsilon \sqrt{8 \pi^{2} \Delta_{\mathcal{R}}^{2}}} \mathcal{B}_{0, \mathrm{dr}} \sim\left(3 \times 10^{5}\right) \mathcal{B}_{0, \mathrm{dr}},
$$

where we have used the estimate $\epsilon \sim 0.01$ and the measured value $\Delta_{\mathcal{R}} \sim 3 \times 10^{-5}$.

Thus, the dominant non-Gaussianity for initial states with $N_{\text {occ }} \sim \mathcal{A}_{I I I} \sim 1$ is due to the three point function of the initial state. This is easy to understand: a nonzero bispectrum is obtained from an initial three point function from just the linear evolution, without requiring any nonlinearities. The contributions to the bispectrum from the initial two and four point functions, on the other hand, are suppressed since they require the nonlinearities in the dynamics. The enhancement factor of $M_{p} /(H \sqrt{\epsilon})$ in Eq. (6.3) was previously obtained in a special case by Agarwal et al. [26]. Note that this contribution to the bispectrum vanishes identically for Gaussian initial states, as studied in many previous investigations [27-33, 36, 37].

The result (6.4) could yield a large bispectrum, detectable if $\mathcal{A}_{I I I} \gtrsim 10^{-3}$. Assuming that $\mathcal{A}_{I I I} \sim N_{\text {occ }}$, the bispectrum could be detectable even for initial occupation numbers small compared to unity, and $N_{\text {occ }} \gtrsim 1$ is compatible with the backreaction constraint (3.21) for suitable values of the spectral index $n$ and bandwidth $\chi^{4}$.

It is not possible to give a generic prediction for the shape dependence of this dominant piece $\mathcal{B}_{I I I}$ of the bispectrum, since the shape dependence is just inherited from that of the initial state. Different scenarios for the origin of the non-vacuum initial state will yield different shape dependences.

\subsection{Initial states with vanishing three point function}

We now specialize to initial states for which the three point function is small or vanishing, for example Gaussian states [27-33, 36, 37] or statistical mixtures of mode occupation number eigenstates [25]. For such states $\mathcal{B}_{I I I}$ vanishes, and we argued above that when $k_{1} \sim k_{2} \sim k_{3}$ and $N_{\text {occ }} \sim 1$ we have

$$
\mathcal{B}_{I I} \sim \mathcal{B}_{I V} \sim \mathcal{B}_{0, \mathrm{dr}}
$$

so that the non-Gaussianity is small in this regime. Nevertheless it is still possible that $\mathcal{B}_{I I}$ and/or $\mathcal{B}_{I V}$ can be large in various limits where $k_{1} \sim k_{2} \sim k_{3}$ is violated or $N_{\text {occ }} \gg 1$. It is not possible to estimate how $\mathcal{B}_{I V}$ scales in such limits, since it depends on unknown properties of the connected part of the initial four point function. Therefore in the remainder of this section we will restrict attention to states for which the bispectrum is dominated by $\mathcal{B}_{I I}$.

\subsubsection{Large occupation number regime}

Let us first consider the possibilities for an enhanced bispectrum in the regime $N_{\text {occ }} \gg 1$, assuming for simplicity that $k_{1} \sim k_{2} \sim k_{3} \sim k$. In this regime the vacuum bispectrum (5.4) scales as $\mathcal{B}_{0, \mathrm{dr}} \sim H^{4} /\left(M_{p}^{4} \epsilon k^{6}\right)$, and from Eq. (5.6) we obtain

$$
\mathcal{B}_{I I} / \mathcal{B}_{0, \mathrm{dr}} \sim\left[N_{\mathrm{occ}}(k) \quad \& \quad N_{\mathrm{occ}}^{2}(k)\right] .
$$

\footnotetext{
${ }^{4}$ On the other hand $N_{\text {occ }} \gtrsim 1$ would give order-unity corrections to the power spectrum (4.6). This would disagree with observations unless the corrected spectrum is nearly scale invariant, which would be a fine tuning.
} 
Here the notation inside the square brackets means that there are two types of term that arise, terms proportional to $N_{\text {occ }}\left(k_{1}\right) \sim \mathcal{F}_{1}\left(k_{1}\right) \sim \mathcal{F}_{2}\left(k_{1}\right) \sim N_{\text {occ }}\left(k_{2}\right) \sim N_{\text {occ }}(k)$, and terms proportional to the products $N_{\text {occ }}\left(k_{1}\right) N_{\text {occ }}\left(k_{2}\right) \sim N_{\text {occ }}(k)^{2}$.

We see from Eq. (6.6) that a bispectrum much larger than the Bunch-Davies bispectrum requires large occupation numbers, for initial states with vanishing three point function. Occupation numbers that exceed unity are compatible with the backreaction constraint. However, as discussed in Sec. 3.2 above, large occupation numbers are only possible over very narrow bandwidths, which is a kind of fine tuning. In addition occupation numbers in excess of unity over a range of wavenumbers is only possible if the range of wavenumbers does not exceed about one and half orders of magnitude, cf. Eq. (3.27) above. Finally, as noted above, occupation numbers $\gtrsim 1$ would give order-unity corrections to the power spectrum (4.6), which would disagree with observations unless the corrected spectrum conspires to be nearly scale invariant. Taken together, these constraints imply that it is difficult for the bispectrum to be significantly larger than the vacuum bispectrum in this regime.

\subsubsection{Limiting shape regimes}

Consider now squeezed triangle configurations, $k_{1} \sim k_{2} \gg k_{3}$. The vacuum bispectrum in this regime is, from Eq. (5.4),

$$
\mathcal{B}_{0, \mathrm{dr}} \sim \frac{H^{4}}{M_{p}^{4} \epsilon k_{1}^{3} k_{3}^{3}} .
$$

The limiting behavior of $\mathcal{B}_{I I}$ in this limit depends on the direction in which the limit is approached, which can be along the edge of the triangle corresponding to elongated configurations, or from the interior of the triangle. If we assume a direction from the interior, the dominant piece of $\mathcal{B}_{I I}$ is given by the amplitude $\hat{\mathcal{A}}_{I I}$. We find from Eq. (5.10) that

$$
\mathcal{B}_{I I} \sim \frac{H^{4}}{M_{p}^{4} \epsilon k_{1}^{3} k_{3}^{3}}\left\{\frac{k_{1}}{k_{3}}\left[N_{\text {occ }}\left(k_{1}\right) \quad \& \quad N_{\text {occ }}\left(k_{1}\right)^{2} \quad \& \quad N_{\text {occ }}\left(k_{1}\right) N_{\text {occ }}\left(k_{3}\right)\right], \quad N_{\text {occ }}\left(k_{3}\right)\right\} .
$$

Here the notation inside the square brackets means that there are three types of term that arise which scale $\propto k_{3}^{-4}$, terms proportional to $N_{\text {occ }}\left(k_{1}\right) \sim N_{\text {occ }}\left(k_{2}\right) \sim \mathcal{F}_{1}\left(k_{1}\right) \sim \mathcal{F}_{2}\left(k_{1}\right)$, terms proportional to the products $N_{\text {occ }}\left(k_{1}\right) N_{\text {occ }}\left(k_{2}\right) \sim N_{\text {occ }}\left(k_{1}\right)^{2}$, and terms proportional to the product $N_{\text {occ }}\left(k_{1}\right) N_{\text {occ }}\left(k_{3}\right)$. Also there are terms proportional to $\mathcal{F}_{1}\left(k_{3}\right) \sim \mathcal{F}_{2}\left(k_{3}\right) \sim N_{\text {occ }}\left(k_{3}\right)$ which scale $\propto k_{3}^{-3}$.

If we now assume that all the occupation numbers are of order unity, following Agullo and Parker [25], then we obtain

$$
\mathcal{B} / \mathcal{B}_{0, \mathrm{dr}} \sim k_{1} / k_{3} \text {. }
$$

Since $k_{1} / k_{3}$ can be as large as $\sim 100$ for modes probed in the CMB, and even larger for modes probed by large scale structure, the estimate (6.9) is a significant enhancement of non-Gaussianity over the standard vacuum result, as previously argued in a special case by Agullo and Parker [25], and by Ganc [32] for the the class of squeezed vacuum states.

However the assumption that all mode occupation numbers are of order unity is quite restrictive and can conflict with the backreaction constraints discussed in Sec. 3.2. We now investigate how large the enhancement factor (6.9) can be assuming the power law model (3.16) is valid over all scales from $k_{3}$ to $k_{1} \sim k_{2}$. We will find that the backreaction constraints imply that the enhancement factor cannot exceed $\sim 200$.

The backreaction constraints will be weakest when $k_{\min } \sim k_{3}$ and $k_{\max } \sim k_{1}$, so we will assume this in what follows. Consider first the first term in square brackets in Eq. (6.8), 
$N_{\text {occ }}\left(k_{1}\right)$, which is constrained by Eq. (3.23). Combining Eqs. (6.9) and (3.23) gives that this contribution to the bispectrum is bounded above by $\mathcal{B}_{I I} / \mathcal{B}_{0, \mathrm{dr}} \lesssim 10^{5} n /\left(\chi^{3}\left(1-\chi^{-n}\right)\right)$, which is of order unity or smaller for $|n| \leq 10$ and $\chi=k_{\max } / k_{\min } \sim k_{1} / k_{3} \gtrsim 10^{2}$. A similar argument applies to the second term in the square brackets in Eq. (6.9). The largest term is the third term, which from Eqs. (3.21), (3.23) and (6.9) is bounded above by

$$
\frac{\mathcal{B}_{I I}}{\mathcal{B}_{0, \mathrm{dr}}} \sim \chi N_{0} N_{1} \lesssim \frac{10^{10} \chi^{n-3}}{f_{n}(\chi)^{2}} \sim \frac{10^{10} \chi^{n-3} n^{2}}{\left(\chi^{n}-1\right)^{2}} \lesssim \frac{10^{10}}{\chi^{3}(\ln \chi)^{2}}
$$

where we have used the fact that the maximum of the function of $n$ is achieved at $n=0$. The upper bound (6.10) can be quite large for $\chi \sim 1$, but that regime corresponds to measuring the bispectrum over a narrow range of scales, and choosing the initial state to populate only those scales, which would be a considerable fine tuning. The the upper bound (6.10) is smaller than $\chi$, invalidating the estimate (6.9), when $\chi \gtrsim 200$.

We now turn to a discussion of the the edge of the triangle corresponding to elongated configurations, i.e. the line $k_{1}=k_{2}+k_{3}$. There is an enhancement of the bispectrum for these configurations. For the special case of squeezed states (cf. Sec. 6.3 below), this enhancement was previously pointed out by Chen et. al. [57] and Holman and Tolley [29], and studied in more detail by Agullo and Shandera [33]. The enhancement arises from the four terms in Eq. (5.10) in which one of the arguments of the function $r(a, b)$ is $\pm \tilde{k}_{1}$. Normally the phases $k \tau_{0}$ in the formula (5.12) for $r(a, b)$ have absolute values which are large compared to unity, since we have assumed that all the modes are inside the horizon at the initial time, $k_{i}\left|\tau_{0}\right| \gg 1$. Therefore the function $r$ scales as $r \sim 1 / k$. However, for elongated configurations, $\tilde{k}_{1} \rightarrow 0$ from Eq. (5.11), and so for example the function $r\left(\tilde{k}_{1},-\tilde{k}_{2}\right)$ scales as $\sim\left|\tau_{0}\right|$, enhanced by a factor $k\left|\tau_{0}\right| \gg 1$. We find from Eqs. (5.10) and (5.12) that in the elongated limit ${ }^{5}$

$$
\frac{\mathcal{B}_{I I}}{\mathcal{B}_{0, \mathrm{dr}}} \sim\left|\tau_{0}\right| k_{1} W\left[k_{1}\left|\tau_{0}\right|\left(x_{2}+x_{3}-1\right)\right]\left[\begin{array}{lll}
N_{\mathrm{occ}} & \& & N_{\mathrm{occ}}^{2}
\end{array}\right]
$$

where $W(x)=\left(1-e^{-i x}\right) /(i x)$. The factor $W\left[k_{1}\left|\tau_{0}\right|\left(x_{2}+x_{3}-1\right)\right]$ goes to unity along the edge $k_{2}+k_{3}=k_{1}$ of the triangle.

How large can the enhancement factor (6.11) be? The factors of occupation number are limited by the backreaction constraint, just as discussed above for squeezed configurations. The function $W$ is of order unity or smaller. The factor $k_{1}\left|\tau_{0}\right|$ can apparently be arbitrarily large, since the initial conformal time $\tau_{0}$ can be chosen to be as early as desired. However, this large $\tau_{0}$ divergence is only apparent, it is an $\operatorname{artifact}^{6}$ of how we have chosen to parameterize the initial state, since it diverges as $\tau_{0} \rightarrow-\infty$. If we specify the initial state at some time $\tau_{0}$, then changing the initial time to some earlier time $\tau_{1}$ will have no affect on the bispectrum if we choose the initial state at $\tau_{1}$ to be that obtained from the initial state at $\tau_{0}$ by evolving backwards in time from $\tau_{0}$ to $\tau_{1}$. Our simple power law model (3.15) and (3.16) for the initial state is not invariant under time evolution when interactions are included, which is the reason for the $\tau_{0}$ dependence of the bispectrum (5.3). To avoid this spurious time dependence, we will choose the value of $\tau_{0}$ to be the latest possible time for which all the modes under consideration are inside the horizon. This choice gives $\left|\tau_{0}\right| \sim 1 / k_{\min }$, so $\left|\tau_{0}\right| k_{1} \sim\left|\tau_{0}\right| k_{\max } \sim k_{\max } / k_{\min } \sim \chi$. With this choice the enhancement factor (6.11) for elongated configurations is of the same order as the enhancement factor (6.10) for squeezed configurations approached from the interior of the triangle.

\footnotetext{
${ }^{5}$ The same estimate also applies to the squeezed limit approached along the edge of the triangle, i.e. via elongated configurations.

${ }^{6}$ This aspect was missed in the analysis of Chen et. al. [57] and Holman and Tolley [29].
} 


\subsubsection{Observational constraints}

We now discuss observational constraints on the class of states under discussion in this section, where the bispectrum is dominated by the initial two point function. As discussed above it is not possible to make detailed predictions for the cases where the bispectrum is dominated by the initial three or four point functions.

For simplicity we make the following assumptions and specializations. We assume that the functions $\mathcal{F}_{1}$ and $\mathcal{F}_{2}$ are real and satisfy $\mathcal{F}_{1}=\mathcal{F}_{2}=N_{\text {occ }}$. For the occupation number $N_{\text {occ }}$ we use the model (3.16) specialized to $n=4$. Then the result (5.6) for the bispectrum simplifies to

$$
\mathcal{B}_{I I} \approx \frac{18}{5} \mathcal{P}^{2}(k)\left(\frac{20}{3} N_{0}^{2} \epsilon\right) \frac{1}{3 k^{3}}\left[\left(k_{1}^{2} k_{2}^{2}+k_{3}^{2} k_{1}^{2}+k_{3}^{2} k_{2}^{2}\right) \sum_{i=1}^{3} \frac{1-\cos \tilde{k}_{i} \tau_{0}}{\tilde{k}_{i}}\right],
$$

where we have omitted terms proportional to $\left(1-\cos k_{t} \tau_{0}\right) / k_{t}$ by virtue of being subdominant to other terms in triangular regimes of interest. Also given our specialization $\mathcal{F}_{1}=\mathcal{F}_{2}$, the power spectrum above is just the Bunch-Davies power spectrum introduced in Sec. 4. From Eq. (6.12) and the definition of $f_{\mathrm{NL}}$ provided in Eq. (6.1) we have

$$
f_{\mathrm{NL}}=\frac{20}{3} N_{0}^{2} \epsilon .
$$

The square bracket on the right of Eq. (6.12) includes the shape functions

$$
\mathcal{S}_{\mathrm{NBD}}^{1} \equiv \frac{1}{2} k_{1}^{2}\left(k_{2}^{2}+k_{3}^{2}\right) \frac{1-\cos \tilde{k_{1}} \tau_{0}}{\tilde{k_{1}}}+(\text { cyclic perm. })
$$

and

$$
\mathcal{S}_{\mathrm{NBD}}^{2} \equiv\left(k_{2}^{2} k_{3}^{2}\right) \frac{1-\cos \tilde{k_{1}} \tau_{0}}{\tilde{k_{1}}}+(\text { cyclic perm. })
$$

which were first introduced as shape function ansatzs by Parker and Agullo[25]. Here "NBD" stands for non Bunch-Davies. They have been analyzed by the Planck collaboration [8], who obtained upper bounds on the corresponding $f_{\mathrm{NL}}$ parameters which they define as

$$
\mathcal{B}_{\mathrm{NBD}}=\frac{2}{k^{3}} \mathcal{P}^{2}(k) f_{\mathrm{NL}}^{\mathrm{NBDi}} \mathcal{S}_{\mathrm{NBD}}^{i}
$$

The parameter $f_{\mathrm{NL}}^{\mathrm{NBD} 1}$ has a shape that peaks in squeezed configurations, while the parameter $f_{\mathrm{NL}}^{\mathrm{NBD} 2}$ has a shape that peaks in folded configurations. Comparing our bispectrum in Eq. (6.12) to the Bunch-Davies bispectrum ansatz in Eqs. (6.14) and (6.15) we find

$$
f_{\mathrm{NL}}^{\mathrm{NBD} 1}=\frac{6}{5} f_{\mathrm{NL}}, \quad f_{\mathrm{NL}}^{\mathrm{NBD} 2}=\frac{27}{20} f_{\mathrm{NL}} .
$$

The Planck collaboration's upper bounds are given in Table 11 of Ref. [8] as

$$
f_{\mathrm{NL}}^{\mathrm{NBD} 1}=31 \pm 19, \quad f_{\mathrm{NL}}^{\mathrm{NBD} 2}=0.8 \pm 0.2 .
$$

The corresponding constraint on the initial occupation number $N_{0}$ obtained from $f_{\mathrm{NL}}^{\mathrm{NBD} 2}$ is more stringent. Specifically, for $\epsilon \sim 0.01$, an occupation number $N_{0} \approx 3$ is obtained which is consistent with the bounds (6.18). This value is however large compared to limits from backreaction or constancy of power spectrum considerations. In the same way, the Planck data does not strongly constrain the bandwidth $\chi$. 


\subsection{Example: multimode squeezed initial states}

An example of a class of initial states to which our analysis applies are multimode squeezed states or generalized vacuum states. These are defined by considering mode operators $\hat{B}_{\vec{k}}$ which are related to the operators $\hat{A}_{\vec{k}}$ of the mode expansion (2.10) by a linear transformation of the form

$$
\left(\begin{array}{c}
\hat{B}_{\vec{k}} \\
\hat{B}_{-\vec{k}}^{\dagger}
\end{array}\right)=\left(\begin{array}{cc}
\alpha^{*}(k) & -\beta^{*}(k) \\
-\beta(k) & \alpha(k)
\end{array}\right)\left(\begin{array}{c}
\hat{A}_{\vec{k}} \\
\hat{A}_{-\vec{k}}^{\dagger}
\end{array}\right),
$$

where $|\alpha(k)|^{2}-|\beta(k)|^{2}=1$. The squeezed state at the initial time $\tau_{0}$ is then the state that is annihilated by the operators $\hat{B}_{\vec{k}}$. This is the class of states that would be produced by starting at an earlier time with a Bunch-Davies vacuum, and then evolving through a homogeneous isotropic expansion phase with a single scalar field. Non-Gaussianities produced by this class of states have been studied in Refs. [27-35].

For this class of states, we can compute the free functions $\mathcal{F}_{i}, \mathcal{H}_{i}$ and $\Gamma_{i}$ that parameterize the initial state by inserting the definition (6.19) into Eqs. (3.1) - (3.8). This gives

$$
\mathcal{H}_{i}=\Gamma_{i}=0,
$$

and

$$
\mathcal{F}_{2}(\vec{k})=|\beta(k)|^{2}, \quad \mathcal{F}_{1}(\vec{k})=\alpha(k) \beta(k)^{*} .
$$

Inserting these functions into our expression (5.6) for the bispectrum reproduces the result obtained previously by Ganc [32], and earlier in a certain limit by Chen et al. [57]. Similarly, inserting these functions into our expression (4.6) for the leading order corrections to the power spectrum reproduces previous estimates of the effects of trans-Planckian physics by Easther et. al. [47], for their choice of Bogolubov coefficients

$$
\alpha=1+x \frac{H}{\Lambda}+\mathcal{O}\left(\frac{H^{2}}{\Lambda^{2}}\right), \quad \beta=y \frac{H}{\Lambda}+\mathcal{O}\left(\frac{H^{2}}{\Lambda^{2}}\right),
$$

where $\Lambda$ is a cutoff.

\section{Acknowledgments}

We thank Liam McAllister for helpful discussions. This research was supported in part by NSF grants PHY-1068541 and PHY-0968820 and by NASA grant NNX11AI95G.

\section{A Free functions for the dressed vacuum state}

In this appendix we compute the free functions $\mathcal{F}_{i}, \mathcal{H}_{i}$ and $\Gamma_{i}$ that characterize the initial state, for the ground state of the interacting theory, the dressed vacuum.

We are interested in evaluating the initial two point, three point and four point functions. The general $n$-point function is

$$
\left\langle\Omega\left|\hat{A}_{\vec{k}_{1}}^{\alpha} \ldots \hat{A}_{\vec{k}_{n}}^{\delta}\right| \Omega\right\rangle
$$

where $|\Omega\rangle$ is the dressed vacuum state. Here the indices $\alpha, \beta$ etc. can be 0 or 1 ; the notation is that $\hat{A}^{\alpha}$ means $\hat{A}$ for $\alpha=0$ and $\hat{A}^{\dagger}$ for $\alpha=1$. Note that the $n$-point function (A.1) is time dependent. In the Schrödinger picture the state $|\Omega\rangle$ is time independent while the operators $\hat{A}_{\vec{k}}$ are time dependent, while in the interaction picture the converse is true. We will evaluate this quantity at the initial conformal time $\tau_{0}$. 
As usual we can evolve expectation values in the dressed vacuum by evolving starting with the free vacuum at early times, with the early time displaced slightly into the complex plane [52, 58]:

$$
\left\langle\Omega\left|\hat{A}_{\vec{k}_{1}}^{\alpha} \ldots \hat{A}_{\vec{k}_{n}}^{\delta}\right| \Omega\right\rangle=\lim _{\tau \rightarrow-\infty(1-i \epsilon)}\left\langle 0\left|\hat{U}\left(\tau_{0}, \tau\right)^{\dagger} \hat{A}_{\vec{k}_{1}}^{\alpha} \ldots \hat{A}_{\vec{k}_{n}}^{\delta} \hat{U}\left(\tau_{0}, \tau\right)\right| 0\right\rangle .
$$

Here $\hat{U}\left(\tau_{0}, \tau\right)$ is the unitary evolution operator that maps interaction picture states at conformal time $\tau$ to conformal time $\tau_{0}$, given by

$$
\hat{U}\left(\tau_{0}, \tau\right)=T \exp \left\{-i \int_{\tau}^{\tau_{0}} d \tau^{\prime} \hat{H}_{\mathrm{int}}^{I}\left(\tau^{\prime}\right)\right\}
$$

Also in Eq. (A.2) the operators $\hat{A}_{\vec{k}}$ are interaction picture operators; we drop the I denoting interaction picture from now on. Expanding to leading order in the interaction gives

$$
\left\langle\Omega\left|\hat{A}_{\vec{k}_{1}}^{\alpha} \ldots \hat{A}_{\vec{k}_{n}}^{\delta}\right| \Omega\right\rangle=\left\langle 0\left|\hat{A}_{\vec{k}_{1}}^{\alpha} \ldots \hat{A}_{\vec{k}_{n}}^{\delta}\right| 0\right\rangle+i \int_{-\infty(1-i \epsilon)}^{\tau_{0}} d \tau\left\langle 0\left|\left[\hat{H}_{\mathrm{int}}(\tau), \hat{A}_{\vec{k}_{1}}^{\alpha} \ldots \hat{A}_{\vec{k}_{n}}^{\delta}\right]\right| 0\right\rangle .
$$

Next, we use the explicit formula (2.17) for the interaction Hamiltonian. Since this interaction Hamiltonian is trilinear in the fields, and since the vacuum expectation value of the product of an odd number of creation or annihilation operators vanishes, it follows from Eq. (A.4) that all of the free functions (3.1) and (3.3) vanish. The only possible non-vanishing free functions are $\mathcal{H}_{1}$ and $\mathcal{H}_{2}$, defined by Eq. (3.2). We now evaluate $\mathcal{H}_{1}^{0, d r}$ to leading order:

$$
\begin{aligned}
\left\langle\Omega\left|\hat{A}_{\vec{k}_{1}} \hat{A}_{\vec{k}_{2}} \hat{A}_{\vec{k}_{3}}\right| \Omega\right\rangle= & (2 \pi)^{3} \delta\left(\vec{k}_{1}+\vec{k}_{2}+\vec{k}_{3}\right) \mathcal{H}_{1}^{0, \mathrm{dr}}\left(\vec{k}_{1}, \vec{k}_{2}\right) \\
= & i \int_{-\infty(1-i \epsilon)}^{\tau_{0}} d \tau\left\langle 0\left|\left[\hat{H}_{\mathrm{int}}, \hat{A}_{\vec{k}_{1}} \hat{A}_{\vec{k}_{2}} \hat{A}_{\vec{k}_{3}}\right]\right| 0\right\rangle \\
= & -\frac{\sqrt{\epsilon} H}{2 M_{p}} \int d \tau \int \frac{d^{3} k^{\prime}}{(2 \pi)^{3}} \int \frac{d^{3} p}{(2 \pi)^{3}} \frac{\sqrt{k^{\prime}\left|\vec{p}-\vec{k}^{\prime}\right| p}}{p^{2}}\langle 0|\left[\left(\hat{A}_{\vec{k}^{\prime}} e^{-i \tau k^{\prime}}-\hat{A}_{-\vec{k}^{\prime}}^{\dagger} e^{i \tau k^{\prime}}\right)\right. \\
& \left.\left(\hat{A}_{\vec{p}-\vec{k}^{\prime}} e^{-i \tau\left|\vec{p}-\vec{k}^{\prime}\right|}-\hat{A}_{\vec{p}-\vec{k}^{\prime}}^{\dagger} e^{i \tau\left|\vec{p}-\vec{k}^{\prime}\right|}\right)\left(\hat{A}_{-\vec{p}} e^{-i \tau p}-\hat{A}_{\vec{p}}^{\dagger} e^{i \tau p}\right), \hat{A}_{\vec{k}_{1}} \hat{A}_{\vec{k}_{2}} \hat{A}_{\vec{k}_{3}}\right]|0\rangle \\
= & -\frac{\sqrt{\epsilon} H}{2 M_{p}} \int_{-\infty(1-i \epsilon)}^{\tau_{0}} d \tau \int \frac{d^{3} k^{\prime}}{(2 \pi)^{3}} \int \frac{d^{3} p}{(2 \pi)^{3}} \frac{\sqrt{k^{\prime}\left|\vec{p}-\vec{k}^{\prime}\right| p}}{p^{2}} \\
& \times\left\langle 0\left|\hat{A}_{\vec{k}_{1}} \hat{A}_{\vec{k}_{2}} \hat{A}_{\vec{k}_{3}} \hat{A}_{-\vec{k}^{\prime}}^{\dagger} \hat{A}_{\vec{p}-\vec{k}^{\prime}}^{\dagger} \hat{A}_{\vec{p}}^{\dagger}\right| 0\right\rangle e^{i \tau\left(k^{\prime}+\left|\vec{p}-\vec{k}^{\prime}\right|+p\right)} .
\end{aligned}
$$

Here we have used the interaction Hamiltonian (2.17), the mode expansion (2.18) and the asymptotic form (2.14) of the mode functions. Simplifying Eq. (A.5) gives

$$
\mathcal{H}_{1}^{0, \mathrm{dr}}\left(\vec{k}_{1}, \vec{k}_{2}\right)=i \frac{H}{M_{p}} \sqrt{\epsilon} \frac{\left(k_{1} k_{2} k_{3}\right)^{1 / 2}}{k_{t}} \sum_{i=1}^{3} \frac{1}{k_{i}^{2}} e^{i \tau_{0} k_{t}},
$$

where $\vec{k}_{3}=-\vec{k}_{1}-\vec{k}_{2}$ and $k_{t}=k_{1}+k_{2}+k_{3}$. A similar calculation shows that $\mathcal{H}_{2}^{0, \mathrm{dr}}$ vanishes.

We note that there is no contribution to these computations from the field redefinition (2.15), since the operators $\hat{A}_{\vec{k}}$ are defined in terms of the mode expansion (2.18) of the redefined field operator $\hat{\mathcal{R}}_{c}$. 


\section{B Explicit calculation of the bispectrum}

We fix values of $\vec{k}_{1}, \vec{k}_{2}$ and $\vec{k}_{3}$, all of whom we assume to be nonvanishing. As discussed near Eq. (5.2), there are two contributions to bispectrum, one from the field redefinition (2.15) and one from the interaction Hamiltonian. We now compute these two contributions in turn.

\section{B.1 Field redefinition contribution}

We substitute the field redefinition (2.15) into the first term on the right hand side of Eq. (5.2). We neglect the second term in Eq. (5.2) for the moment; that term will be treated in Sec. B. 2 below. The result is

$$
\begin{aligned}
\left\langle\hat{\mathcal{R}}_{\vec{k}_{1}}^{I} \hat{\mathcal{R}}_{\vec{k}_{2}}^{I} \hat{\mathcal{R}}_{\vec{k}_{3}}^{I}\right\rangle & =\left\langle\hat{\mathcal{R}}_{c, \vec{k}_{1}}^{I} \hat{\mathcal{R}}_{c, \vec{k}_{2}}^{I} \hat{\mathcal{R}}_{c, \vec{k}_{3}}^{I}\right\rangle \\
& +\left(\frac{1}{2} \frac{\ddot{\phi}}{\phi H}+\frac{1}{8 M_{p}^{2}} \frac{\dot{\phi}^{2}}{H^{2}}\right)\left(\int \frac{d^{3} p}{(2 \pi)^{3}}\left\langle\hat{\mathcal{R}}_{c, \vec{k}_{1}}^{I} \hat{\mathcal{R}}_{c, \vec{k}_{2}}^{I} \hat{\mathcal{R}}_{c, \vec{p}}^{I} \hat{\mathcal{R}}_{c, \vec{k}_{3}-\vec{p}}^{I}\right\rangle+\text { cyclic perms }\right) \\
& +\frac{\dot{\phi}^{2}}{4 M_{p}^{2} H^{2}}\left(\frac{1}{k_{3}^{2}} \int \frac{d^{3} p}{(2 \pi)^{3}}\left\langle\hat{\mathcal{R}}_{c, \vec{k}_{1}}^{I} \hat{\mathcal{R}}_{c, \vec{k}_{2}}^{I} \hat{\mathcal{R}}_{c, \vec{p}}^{I} \hat{\mathcal{R}}_{c, \vec{k}_{3}-\vec{p}}^{I}\right\rangle\left(\vec{k}_{3}-\vec{p}\right)^{2}+\text { cyclic perms }\right) .
\end{aligned}
$$

Using the mode expansion (2.18), the asymptotic form (2.14) of the mode functions, the decomposition (3.10) of the function $\mathcal{H}_{1}$, and noting that one point functions vanish because of our homogeneity assumption, we find for the first term in Eq. (B.1)

$$
\left\langle\hat{\mathcal{R}}_{c, \vec{k}_{1}}^{I} \hat{\mathcal{R}}_{c, \vec{k}_{2}}^{I} \hat{\mathcal{R}}_{c, \vec{k}_{3}}^{I}\right\rangle=(2 \pi)^{3} \delta\left(\vec{k}_{1}+\vec{k}_{2}+\vec{k}_{3}\right)\left[\mathcal{B}_{I I I}\left(\vec{k}_{1}, \vec{k}_{2}, \vec{k}_{3}\right)+\mathcal{B}_{I I I}\left(\vec{k}_{1}, \vec{k}_{2}, \vec{k}_{3}\right)^{0, \mathrm{dr}}\right] .
$$

Here

$$
\begin{aligned}
\mathcal{B}_{I I I}\left(\vec{k}_{1}, \vec{k}_{2}, \vec{k}_{3}\right)= & -\frac{i H^{3}}{8 M_{p}^{3} \epsilon^{\frac{3}{2}}} \frac{1}{\left(k_{1} k_{2} k_{3}\right)^{\frac{3}{2}}}\left[\hat{\mathcal{H}}_{1}\left(\vec{k}_{1}, \vec{k}_{2}\right)-\hat{\mathcal{H}}_{1}\left(-\vec{k}_{1},-\vec{k}_{2}\right)^{*}-\mathcal{H}_{2}\left(\vec{k}_{1}, \vec{k}_{2}\right)\right. \\
& +\mathcal{H}_{2}\left(-\vec{k}_{1},-\vec{k}_{2}\right)^{*}-\mathcal{H}_{2}\left(\vec{k}_{1}, \vec{k}_{3}\right)+\mathcal{H}_{2}\left(-\vec{k}_{1},-\vec{k}_{3}\right)^{*}-\mathcal{H}_{2}\left(\vec{k}_{2}, \vec{k}_{3}\right) \\
& \left.+\mathcal{H}_{2}\left(-\vec{k}_{2},-\vec{k}_{3}\right)^{*}\right]
\end{aligned}
$$

and

$$
\begin{aligned}
\mathcal{B}_{I I I}\left(\vec{k}_{1}, \vec{k}_{2}, \vec{k}_{3}\right)^{0, \mathrm{dr}}= & -\frac{i H^{3}}{8 M_{p}^{3} \epsilon^{\frac{3}{2}}} \frac{1}{\left(k_{1} k_{2} k_{3}\right)^{\frac{3}{2}}}\left[\mathcal{H}_{1}^{0, \mathrm{dr}}\left(\vec{k}_{1}, \vec{k}_{2}\right)-\mathcal{H}_{1}^{0, \mathrm{dr}}\left(-\vec{k}_{1},-\vec{k}_{2}\right)^{*}\right] \\
& =\frac{H^{4}}{4 M_{p}^{4}} \frac{\epsilon}{k_{1} k_{2} k_{3}} \sum_{i=0}^{3} \frac{1}{k_{i}^{2} k_{t}} \cos \left(\tau_{0} k_{t}\right),
\end{aligned}
$$

where we used the expression (A.6) for $\mathcal{H}_{1}^{0, \mathrm{dr}}$. 
We can similarly evaluate the products of four field operators that appear in Eq. (B.1). We find that $\left\langle\hat{\mathcal{R}}_{\vec{k}_{1}} \hat{\mathcal{R}}_{\vec{k}_{2}} \hat{\mathcal{R}}_{\vec{p}} \hat{\mathcal{R}}_{\vec{k}_{3}-\vec{p}}\right\rangle$ evaluates to

$$
\begin{aligned}
& (2 \pi)^{6} \delta\left(\vec{k}_{1}+\vec{k}_{2}+\vec{k}_{3}\right) \frac{H^{4}}{16 M_{p}^{4} \epsilon^{2}} \frac{1}{\left(k_{1} k_{2} p\left|\vec{k}_{3}-\vec{p}\right|\right)^{\frac{3}{2}}}\left[\mathcal{G}_{1}\left(\vec{k}_{1}, \vec{k}_{2}, \vec{p}\right)-\mathcal{G}_{2}\left(\vec{k}_{3}-\vec{p}, \vec{k}_{1}, \vec{k}_{2}\right)-\mathcal{G}_{2}\left(\vec{p}, \vec{k}_{1}, \vec{k}_{2}\right)\right. \\
& -\mathcal{G}_{2}\left(\vec{k}_{2}, \vec{k}_{1}, \vec{p}\right)-\mathcal{G}_{2}\left(-\vec{k}_{1},-\vec{k}_{2},-\vec{p}\right)^{*}-\mathcal{G}_{2}\left(\vec{k}_{1}, \vec{k}_{2}, \vec{p}\right)-\mathcal{G}_{2}\left(-\vec{k}_{2},-\vec{k}_{1},-\vec{p}\right)^{*}-\mathcal{G}_{2}\left(-\vec{p},-\vec{k}_{1},-\vec{k}_{2}\right)^{*} \\
& -\mathcal{G}_{2}\left(\vec{p}-\vec{k}_{3},-\vec{k}_{1},-\vec{k}_{2}\right)^{*}+\mathcal{G}_{3}\left(\vec{p}, \vec{k}_{3}-\vec{p}, \vec{k}_{1}\right)+\mathcal{G}_{3}\left(\vec{k}_{2}, \vec{k}_{3}-\vec{p}, \vec{k}_{1}\right)+\mathcal{G}_{3}\left(\vec{k}_{2}, \vec{p}, \vec{k}_{1}\right)+\mathcal{G}_{3}\left(\vec{k}_{1}, \vec{k}_{3}-\vec{p}, \vec{k}_{2}\right) \\
& +\mathcal{G}_{3}\left(\vec{k}_{1}, \vec{p}, \vec{k}_{2}\right)+\mathcal{G}_{3}\left(\vec{k}_{1}, \vec{k}_{2}, \vec{p}\right)+\mathcal{G}_{1}\left(-\vec{k}_{1},-\vec{k}_{2},-\vec{p}\right)^{*} \\
& +\delta\left(\vec{p}+\vec{k}_{1}\right)\left\{-2 \operatorname{Re} \mathcal{F}_{1}\left(\vec{k}_{1}\right)-2 \operatorname{Re} \mathcal{F}_{1}\left(\vec{k}_{2}\right)+\mathcal{F}_{2}\left(-\vec{k}_{2}\right)+\mathcal{F}_{2}\left(-\vec{k}_{1}\right)+\mathcal{F}_{2}\left(\vec{k}_{2}\right)+\mathcal{F}_{2}\left(\vec{k}_{1}\right)\right\} \\
& +\delta\left(\vec{p}+\vec{k}_{2}\right)\left\{-2 \operatorname{Re} \mathcal{F}_{1}\left(\vec{k}_{1}\right)-2 \operatorname{Re} \mathcal{F}_{1}\left(\vec{k}_{2}\right)+\mathcal{F}_{2}\left(-\vec{k}_{2}\right)+\mathcal{F}_{2}\left(-\vec{k}_{1}\right)+\mathcal{F}_{2}\left(\vec{k}_{2}\right)+\mathcal{F}_{2}\left(\vec{k}_{1}\right)\right\} \\
& \left.+\delta\left(\vec{p}+\vec{k}_{1}\right)+\delta\left(\vec{p}+\vec{k}_{2}\right)\right] .
\end{aligned}
$$

We now substitute this expression together with the expression (B.2) back into Eq. (B.1), and then into Eq. (5.2), and then simplify using the decompositions (3.8). This yields the following pieces of our expression (5.3) for the bispectrum: a part of the vacuum term $\mathcal{B}_{0, \mathrm{dr}}$, the term $\mathcal{B}_{I I I}$, the pieces $\mathcal{A}_{I I}$ and $\tilde{\mathcal{A}}_{I I}$ of the term $\mathcal{B}_{I I}$, and the pieces $\mathcal{A}_{I V}$ and $\tilde{\mathcal{A}}_{I V}$ of the term $\mathcal{B}_{I V}$. It also generates the additional term (B.4) which will be canceled by a term obtained in the next subsection.

\section{B.2 Commutator contribution}

We now evaluate the second term in Eq. (5.2). For this term the effect of the field redefinition is of subleading order and can be neglected. Using the interaction Hamiltonian (2.17), the mode expansion (2.18), and the asymptotic form (2.14) of the mode functions we find that this term evaluates to

$$
\begin{aligned}
& -\int_{\tau_{0}}^{0} d \tau^{\prime} \frac{i H^{4}}{16 \epsilon M_{p}^{4}}\left(\frac{1}{k_{1} k_{2} k_{3}}\right)^{\frac{3}{2}} \int \frac{d^{3} p}{(2 \pi)^{3}} \frac{d^{3} k^{\prime}}{(2 \pi)^{3}} \frac{\sqrt{p k^{\prime}\left|\vec{p}-\vec{k}^{\prime}\right|}}{p^{2}}\left\langle\left[\left(\hat{A}_{\vec{k}_{1}}-\hat{A}_{-\vec{k}_{1}}^{\dagger}\right)\left(\hat{A}_{\vec{k}_{2}}-\hat{A}_{-\vec{k}_{2}}^{\dagger}\right)\right.\right. \\
& \times\left(\hat{A}_{\vec{k}_{3}}-\hat{A}_{-\vec{k}_{3}}^{\dagger}\right),\left(\hat{A}_{\vec{k}^{\prime}} e^{-i \tau^{\prime} k^{\prime}}-\hat{A}_{-\vec{k}^{\prime}}^{\dagger} e^{i \tau^{\prime} k^{\prime}}\right)\left(\hat{A}_{\vec{p}-\vec{k}^{\prime}} e^{-i \tau^{\prime}\left|\vec{p}-\vec{k}^{\prime}\right|}-\hat{A}_{\vec{k}^{\prime}-\vec{p}}^{\dagger} e^{i \tau^{\prime}\left|\vec{p}-\vec{k}^{\prime}\right|}\right) \\
& \left.\left.\times\left(\hat{A}_{-\vec{p}} e^{-i \tau^{\prime} p}-\hat{A}_{\vec{p}}^{\dagger} e^{i \tau^{\prime} p}\right)\right]\right\rangle .
\end{aligned}
$$


Using the definitions (3.1) and (3.3) of the free functions $\mathcal{F}_{i}$ and $\mathcal{G}_{i}$, the expression inside the expectation value brackets $\langle\ldots\rangle$ in Eq. (B.6) evaluates to

$$
\begin{aligned}
& (2 \pi)^{9} \delta\left(\vec{p}-\vec{k}_{3}\right) \delta\left(\vec{k}_{1}+\vec{k}_{2}+\vec{p}\right)\left[\left(e^{-i \tau^{\prime}\left(k^{\prime}+\left|\vec{p}-\vec{k}^{\prime}\right|+p\right)}-e^{-i \tau^{\prime}\left(k^{\prime}+\left|\vec{p}-\vec{k}^{\prime}\right|-p\right)}\right)\left(\mathcal{G}_{1}\left(\vec{k}_{1}, \vec{k}_{2}, \vec{k}^{\prime}\right)+\mathcal{G}_{3}\left(\vec{k}_{2}, \vec{k}_{1}, \vec{k}^{\prime}\right)\right)\right. \\
& +\left(e^{-i \tau^{\prime}\left(k^{\prime}-\left|\vec{p}-\vec{k}^{\prime}\right|-p\right)}-e^{-i \tau^{\prime}\left(k^{\prime}-\left|\vec{p}-\vec{k}^{\prime}\right|+p\right)}\right)\left(\mathcal{G}_{2}\left(\vec{p}-\vec{k}^{\prime}, \vec{k}_{1}, \vec{k}_{2}\right)+\mathcal{G}_{2}\left(-\vec{k}^{\prime},-\vec{k}_{1},-\vec{k}_{2}\right)^{*}\right) \\
& +\left(e^{-i \tau^{\prime}\left(-k^{\prime}+\left|\vec{p}-\vec{k}^{\prime}\right|-p\right)}-e^{-i \tau^{\prime}\left(-k^{\prime}+\left|\vec{p}-\vec{k}^{\prime}\right|+p\right)}\right)\left(\mathcal{G}_{2}\left(\vec{k}^{\prime}, \vec{k}_{1}, \vec{k}_{2}\right)+\mathcal{G}_{2}\left(\vec{k}^{\prime}-\vec{p},-\vec{k}_{1},-\vec{k}_{2}\right)^{*}\right) \\
& +\left(e^{-i \tau^{\prime}\left(-k^{\prime}-\left|\vec{p}-\vec{k}^{\prime}\right|+p\right)}-e^{-i \tau^{\prime}\left(-k^{\prime}-\left|\vec{p}-\vec{k}^{\prime}\right|-p\right)}\right)\left(\mathcal{G}_{3}\left(\vec{p}-\vec{k}^{\prime}, \vec{k}^{\prime}, \vec{k}_{1}\right)+\mathcal{G}_{1}\left(-\vec{k}_{1},-\vec{k}_{2},-\vec{p}\right)^{*}\right) \\
& +\left(e^{-i \tau^{\prime}\left(k^{\prime}+\left|\vec{p}-\vec{k}^{\prime}\right|+p\right)}-e^{-i \tau^{\prime}\left(k^{\prime}+\left|\vec{p}-\vec{k}^{\prime}\right|-p\right)}\right)\left(\mathcal{G}_{2}\left(\vec{k}_{1}, \vec{k}_{2}, \vec{k}^{\prime}\right)+\mathcal{G}_{2}\left(\vec{k}_{2}, \vec{k}_{1}, \vec{k}^{\prime}\right)\right) \\
& +\left(e^{-i \tau^{\prime}\left(k^{\prime}-\left|\vec{p}-\vec{k}^{\prime}\right|+p\right)}-e^{-i \tau^{\prime}\left(k^{\prime}-\left|\vec{p}-\vec{k}^{\prime}\right|-p\right)}\right)\left(\mathcal{G}_{3}\left(\vec{p}-\vec{k}^{\prime}, \vec{k}_{1}, \vec{k}_{2}\right)+\mathcal{G}_{3}\left(\vec{p}-\vec{k}^{\prime}, \vec{k}_{2}, \vec{k}_{1}\right)\right) \\
& +\left(e^{-i \tau^{\prime}\left(-k^{\prime}+\left|\vec{p}-\vec{k}^{\prime}\right|+p\right)}-e^{-i \tau^{\prime}\left(-k^{\prime}+\left|\vec{p}-\vec{k}^{\prime}\right|-p\right)}\right)\left(\mathcal{G}_{3}\left(\vec{k}^{\prime}, \vec{k}_{1}, \vec{k}_{2}\right)+\mathcal{G}_{3}\left(\vec{k}^{\prime}, \vec{k}_{2}, \vec{k}_{1}\right)\right) \\
& \left.+\left(e^{-i \tau^{\prime}\left(-k^{\prime}-\left|\vec{p}-\vec{k}^{\prime}\right|-p\right)}-e^{-i \tau^{\prime}\left(-k^{\prime}-\left|\vec{p}-\vec{k}^{\prime}\right|+p\right)}\right)\left(\mathcal{G}_{2}\left(-\vec{k}_{1},-\vec{k}_{2},-\vec{k}^{\prime}\right)^{*}+\mathcal{G}_{2}\left(-\vec{k}_{2},-\vec{k}_{1},-\vec{k}^{\prime}\right)^{*}\right)\right] \\
& +(2 \pi)^{9} \delta\left(\vec{p}-\vec{k}_{3}\right)\left(\delta\left(\vec{k}^{\prime}+\vec{k}_{1}\right) \delta\left(\vec{k}_{2}+\vec{p}-\vec{k}^{\prime}\right)+\delta\left(\vec{k}_{2}+\vec{k}^{\prime}\right) \delta\left(\vec{k}_{1}+\vec{p}-\vec{k}^{\prime}\right)\right) \\
& \times\left[\left(e^{-i \tau^{\prime}\left(-k^{\prime}-\left|\vec{p}-\vec{k}^{\prime}\right|+p\right)}-e^{-i \tau^{\prime}\left(k^{\prime}+\left|\vec{p}-\vec{k}^{\prime}\right|+p\right)}\right) \mathcal{F}_{1}(-\vec{p})+\left(e^{-i \tau^{\prime}\left(k^{\prime}+\left|\vec{p}-\vec{k}^{\prime}\right|-p\right)}-e^{-i \tau^{\prime}\left(-k^{\prime}-\left|\vec{p}-\vec{k}^{\prime}\right|-p\right)}\right) \mathcal{F}_{2}(-\vec{p})\right. \\
& \left.+\left(e^{-i \tau^{\prime}\left(k^{\prime}+\left|\vec{p}-\vec{k}^{\prime}\right|+p\right)}-e^{-i \tau^{\prime}\left(-k^{\prime}-\left|\vec{p}-\vec{k}^{\prime}\right|+p\right)}\right) \mathcal{F}_{2}(\vec{p})^{*}+\left(e^{-i \tau^{\prime}\left(-k^{\prime}-\left|\vec{p}-\vec{k}^{\prime}\right|-p\right)}-e^{-i \tau^{\prime}\left(k^{\prime}+\left|\vec{p}-\vec{k}^{\prime}\right|-p\right)}\right) \mathcal{F}_{1}(\vec{p})^{*}\right] \\
& -2 i(2 \pi)^{9} \delta\left(\vec{p}-\vec{k}_{3}\right)\left(\delta\left(\vec{k}^{\prime}+\vec{k}_{1}\right) \delta\left(\vec{k}_{2}+\vec{p}-\vec{k}^{\prime}\right)+\delta\left(\vec{k}_{2}+\vec{k}^{\prime}\right) \delta\left(\vec{k}_{1}+\vec{p}-\vec{k}^{\prime}\right)\right) \sin \tau^{\prime}\left(k^{\prime}+|\vec{p}-\vec{k}|+p\right) \\
& +\left(\vec{p} \leftrightarrow-\vec{k}^{\prime}\right)+\left(\vec{p} \leftrightarrow \vec{k}^{\prime}-\vec{p}\right) . \\
& + \text { cyclic perms. }
\end{aligned}
$$

We now substitute the expression (B.7) into Eq. (B.6) and then into Eq. (5.2), and then simplify using the decompositions (3.8). This yields the remaining pieces of our expression (5.3) for the bispectrum: the remaining part of the vacuum term $\mathcal{B}_{0, \mathrm{dr}}$, the piece $\hat{\mathcal{A}}_{I I}$ of the term $\mathcal{B}_{I I}$, and the piece $\hat{\mathcal{A}}_{I V}$ of the term $\mathcal{B}_{I V}$. It also generates an additional term, from the time integral of the third last line of Eq. (B.7), which cancels the contribution (B.4).

\section{References}

[1] S. Weinberg, Cosmology. Oxford University Press, 2008.

[2] S. Dodelson, Modern cosmology. 2003.

[3] D. Baumann, TASI Lectures on Inflation, arXiv:0907.5424.

[4] V. Mukhanov, Physical Foundations of Cosmology. Mar., 2001.

[5] Planck collaboration Collaboration, P. Ade et al., Planck 2013 results. XV. CMB power spectra and likelihood, arXiv:1303.5075.

[6] Planck Collaboration, P. Ade et al., Planck 2013 results. XVI. Cosmological parameters, arXiv: 1303.5076.

[7] Planck Collaboration, P. Ade et al., Planck 2013 results. XXII. Constraints on inflation, arXiv:1303.5082. 
[8] Planck Collaboration, P. Ade et al., Planck 2013 Results. XXIV. Constraints on primordial non-Gaussianity, arXiv:1303.5084.

[9] V. Mukhanov and G. Chibisov, Quantum fluctuations and a nonsingular universe, JETP Lett.(Engl. Transl.);(United States) 33 (1981), no. 10.

[10] S. W. Hawking, The development of irregularities in a single bubble inflationary universe, Physics Letters B 115 (Sept., 1982) 295-297.

[11] A. H. Guth and S.-Y. Pi, Fluctuations in the new inflationary universe, Physical Review Letters 49 (Oct., 1982) 1110-1113.

[12] A. Starobinsky, Dynamics of phase transition in the new inflationary universe scenario and generation of perturbations, Phys. Lett. B 117 (1982) 175.

[13] J. M. Bardeen, P. J. Steinhardt, and M. S. Turner, Spontaneous creation of almost scale-free density perturbations in an inflationary universe, Phys. Rev. D 28 (Aug., 1983) 679-693.

[14] E. Komatsu, N. Afshordi, N. Bartolo, D. Baumann, J. R. Bond, E. I. Buchbinder, C. T. Byrnes, X. Chen, D. J. H. Chung, A. Cooray, P. Creminelli, N. Dalal, O. Dore, R. Easther, A. V. Frolov, J. Khoury, W. H. Kinney, L. Kofman, K. Koyama, L. Leblond, J.-L. Lehners, J. E. Lidsey, M. Liguori, E. A. Lim, A. Linde, D. H. Lyth, J. Maldacena, S. Matarrese, L. McAllister, P. McDonald, S. Mukohyama, B. Ovrut, H. V. Peiris, A. Riotto, Y. Rodrigues, M. Sasaki, R. Scoccimarro, D. Seery, A. Sefusatti, K. M. Smith, A. A. Starobinsky, P. J. Steinhardt, F. Takahashi, M. Tegmark, A. J. Tolley, L. Verde, B. D. Wandelt, D. Wands, S. Weinberg, M. Wyman, A. P. S. Yadav, and M. Zaldarriaga, Non-Gaussianity as a Probe of the Physics of the Primordial Universe and the Astrophysics of the Low Redshift Universe, in astro2010: The Astronomy and Astrophysics Decadal Survey, vol. 2010 of ArXiv Astrophysics e-prints, p. 158, 2009. arXiv:0902.4759.

[15] Planck Collaboration, P. Ade et al., Planck 2013 results. XXIII. Isotropy and Statistics of the $C M B$, arXiv: 1303.5083.

[16] C. Pitrou, T. S. Pereira, and J.-P. Uzan, Predictions from an anisotropic inflationary era, JCAP 4 (Apr., 2008) 4, [arXiv:0801.3596].

[17] H.-C. Kim and M. Minamitsuji, An Analytic approach to perturbations from an initially anisotropic universe, JCAP 1103 (2011) 038, [arXiv:1101.0329].

[18] A. Gumrukcuoglu, C. R. Contaldi, and M. Peloso, Inflationary perturbations in anisotropic backgrounds and their imprint on the CMB, JCAP 0711 (2007) 005, [arXiv:0707.4179].

[19] P. K. Samal, R. Saha, P. Jain, and J. P. Ralston, Testing Isotropy of Cosmic Microwave Background Radiation, Mon.Not.Roy.Astron.Soc. 385 (2008) 1718, [arXiv:0708.2816].

[20] P. K. Aluri and P. Jain, Large scale anisotropy due to pre-inflationary phase of cosmic evolution, Mod.Phys.Lett. A27 (2012) 1250014, [arXiv:1108.3643].

[21] A. R. Pullen and M. Kamionkowski, Cosmic Microwave Background Statistics for a Direction-Dependent Primordial Power Spectrum, Phys.Rev. D76 (2007) 103529, [arXiv:0709.1144].

[22] A. Dey and S. Paban, Non-gaussianities in the cosmological perturbation spectrum due to primordial anisotropy, JCAP 4 (Apr., 2012) 39, [arXiv:1106.5840].

[23] A. Dey, E. Kovetz, and S. Paban, Non-Gaussianities in the cosmological perturbation spectrum due to primordial anisotropy II, JCAP 10 (Oct., 2012) 55, [arXiv: 1205.2758].

[24] L. McAllister, S. Renaux-Petel, and G. Xu, A Statistical Approach to Multifield Inflation: Many-field Perturbations Beyond Slow Roll, JCAP 1210 (2012) 046, [arXiv:1207.0317].

[25] I. Agullo and L. Parker, Non-gaussianities and the Stimulated creation of quanta in the inflationary universe, Phys.Rev. D83 (2011) 063526, [arXiv:1010.5766]. 
[26] N. Agarwal, R. Holman, A. J. Tolley, and J. Lin, Effective field theory and non-Gaussianity from general inflationary states, arXiv:1212.1172.

[27] F. Nitti, M. Porrati, and J.-W. Rombouts, Naturalness in cosmological initial conditions, Phys.Rev. D72 (2005) 063503, [hep-th/0503247].

[28] M. Porrati, Effective field theory approach to cosmological initial conditions: Self-consistency bounds and non-Gaussianities, hep-th/0409210.

[29] R. Holman and A. J. Tolley, Enhanced Non-Gaussianity from Excited Initial States, JCAP 0805 (2008) 001, [arXiv:0710.1302].

[30] P. D. Meerburg, J. P. van der Schaar, and P. S. Corasaniti, Signatures of Initial State Modifications on Bispectrum Statistics, JCAP 0905 (2009) 018, [arXiv:0901.4044].

[31] P. D. Meerburg, J. P. van der Schaar, and M. G. Jackson, Bispectrum signatures of a modified vacuum in single field inflation with a small speed of sound, JCAP 1002 (2010) 001, [arXiv:0910.4986].

[32] J. Ganc, Calculating the local-type fNL for slow-roll inflation with a non-vacuum initial state, Phys.Rev. D84 (2011) 063514, [arXiv: 1104.0244].

[33] I. Agullo and S. Shandera, Large non-Gaussian Halo Bias from Single Field Inflation, JCAP 1209 (2012) 007, [arXiv:1204.4409].

[34] A. Ashoorioon, K. Dimopoulos, M. Sheikh-Jabbari, and G. Shiu, Reconciliation of High Energy Scale Models of Inflation with Planck, arXiv:1306.4914.

[35] A. Aravind, D. Lorshbough, and S. Paban, Non-Gaussianity from Excited Initial Inflationary States, JHEP 1307 (2013) 076, [arXiv: 1303.1440].

[36] S. Kundu, Inflation with General Initial Conditions for Scalar Perturbations, JCAP 1202 (2012) 005, [arXiv:1110.4688].

[37] S. Das and S. Mohanty, Non-Gaussianity as a signature of thermal initial condition of inflation, Phys.Rev. D80 (2009) 123537, [arXiv:0908.2305].

[38] A. Ashoorioon and G. Shiu, A Note on Calm Excited States of Inflation, JCAP 1103 (2011) 025, [arXiv: 1012.3392].

[39] K. Goldstein and D. A. Lowe, Initial state effects on the cosmic microwave background and transPlanckian physics, Phys.Rev. D67 (2003) 063502, [hep-th/0208167].

[40] J. Martin and R. H. Brandenberger, The TransPlanckian problem of inflationary cosmology, Phys.Rev. D63 (2001) 123501, [hep-th/0005209].

[41] H. Collins and R. Holman, Trans-Planckian enhancements of the primordial non-Gaussianities, Phys.Rev. D80 (2009) 043524, [arXiv: 0905.4925].

[42] R. Easther, B. R. Greene, W. H. Kinney, and G. Shiu, Inflation as a probe of short distance physics, Phys.Rev. D64 (2001) 103502, [hep-th/0104102].

[43] R. Easther, B. R. Greene, W. H. Kinney, and G. Shiu, Imprints of short distance physics on inflationary cosmology, Phys.Rev. D67 (2003) 063508, [hep-th/0110226].

[44] R. H. Brandenberger and J. Martin, On signatures of short distance physics in the cosmic microwave background, Int.J.Mod.Phys. A17 (2002) 3663-3680, [hep-th/0202142].

[45] R. Brandenberger and P.-M. Ho, Noncommutative space-time, stringy space-time uncertainty principle, and density fluctuations, Phys.Rev. D66 (2002) 023517, [hep-th/0203119].

[46] F. Lizzi, G. Mangano, G. Miele, and M. Peloso, Cosmological perturbations and short distance physics from noncommutative geometry, JHEP 0206 (2002) 049, [hep-th/0203099]. 
[47] R. Easther, B. R. Greene, W. H. Kinney, and G. Shiu, A Generic estimate of transPlanckian modifications to the primordial power spectrum in inflation, Phys.Rev. D66 (2002) 023518, [hep-th/0204129].

[48] U. H. Danielsson, Inflation, holography, and the choice of vacuum in de Sitter space, JHEP 0207 (2002) 040, [hep-th/0205227].

[49] N. Kaloper, M. Kleban, A. E. Lawrence, and S. Shenker, Signatures of short distance physics in the cosmic microwave background, Phys.Rev. D66 (2002) 123510, [hep-th/0201158].

[50] P. Creminelli and M. Zaldarriaga, Single field consistency relation for the 3-point function, JCAP 0410 (2004) 006, [astro-ph/0407059].

[51] T. S. Bunch and P. C. W. Davies, Quantum field theory in de Sitter space - Renormalization by point-splitting, Royal Society of London Proceedings Series A 360 (Mar., 1978) 117-134.

[52] J. M. Maldacena, Non-Gaussian features of primordial fluctuations in single field inflationary models, JHEP 0305 (2003) 013, [astro-ph/0210603].

[53] CMBPol Study Team Collaboration, D. Baumann et al., CMBPol Mission Concept Study: Probing Inflation with CMB Polarization, AIP Conf.Proc. 1141 (2009) 10-120, [arXiv:0811.3919].

[54] R. Flauger, D. Green, and R. A. Porto, On squeezed limits in single-field inflation. Part I, JCAP 1308 (2013) 032, [arXiv: 1303.1430].

[55] P. R. Anderson, C. Molina-París, and E. Mottola, Short distance and initial state effects in inflation: Stress tensor and decoherence, Phys. Rev. D 72 (Aug., 2005) 043515, [0504134].

[56] M. G. Jackson, Integrating out Heavy Fields in Inflation, arXiv:1203.3895.

[57] X. Chen, M.-x. Huang, S. Kachru, and G. Shiu, Observational signatures and non-Gaussianities of general single field inflation, JCAP 0701 (2007) 002, [hep-th/0605045].

[58] M. E. Peskin and D. V. Schroeder, An Introduction to Quantum Field Theory; 1995 ed. (Section 4.2). Westview, Boulder, CO, 1995. 